\title{
Path Integrals and Quantum Mechanics
}

\author{
Martin Sandström \\ Department Of Physics \\ Umeà University \\ Supervisor: Jens Zamanian
}

October 1, 2015

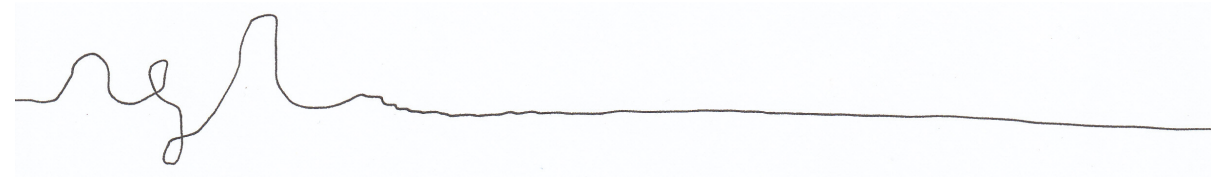

\begin{abstract}
In this thesis we are investigating a different formalism of non-relativistic quantum mechanics called the path integral formalism. It is a generalization of the classical least action principle. The introduction to this subject begins with the construction of the path integral in terms of the idea of probability amplitudes whose absolute square gives the probability of finding a system in a particular state. Then we show that if the Lagrangian is a quadratic form one needs only to calculate the classical action besides from a time-dependent normalization constant to find the explicit expression of the path integral. We look in to the subject of two kinds of slit-experiments: The square slit, the single- and the double-Gaussian slit. Also, the propagator for constrained paths is calculated and applied to the Aharonov-Bohm effect, which shows that the vector potential defined in classical electrodynamics have a physical meaning in quantum mechanics. It is also shown that the path integral formulation is equivalent to the Schrödinger description of quantum mechanics, by deriving the Schrödinger equation from the path integral. Further applications of the path integral are discussed.
\end{abstract}




\section{Contents}

1 Introduction 3

2 Least Action in Classical Mechanics 5

\begin{tabular}{|lll}
3 & Least Action in Quantum Mechanics & 7
\end{tabular}

3.1 Summing over Paths $\ldots \ldots \ldots \ldots \ldots \ldots \ldots \ldots$. . . . . . . . . . . 7

$3.2 \quad$ Propagator with a Quadratic Lagrangian . . . . . . . . . . . . . . . . . . . . . . 8

3.3 Separating the Propagator into Multiple Propagations . . . . . . . . . . . . . . 10

$3.4 \quad$ Writing the full Propagator with a Time-Slicing Method . . . . . . . . . . . . . . . 11

3.5 Orthonormality Condition between Propagators . . . . . . . . . . . . . . . . . . . . 12

3.6 The Relation of the Path Integral to the Schrödinger Equation . . . . . . . . . . . 14

3.7 Obtaining the Hamilton-Jacobi Equation from a Wave Function. . . . . . . . . . . . 15

3.8 The Propagator in terms of Solutions of the Schrödinger Equation . . . . . . . . . 16

$\begin{array}{lll}4 & \text { Applications of the Path Integral } & 16\end{array}$

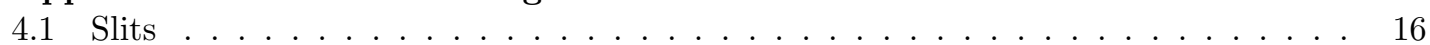

4.1 .1 Single Slit of a Square Type . . . . . . . . . . . . . . . . . . . . . 16

$4.1 .2 \quad$ Single Slit of Gaussian Type $\ldots \ldots \ldots$

4.1 .3 Double Slit of a Gaussian Type . . . . . . . . . . . . . . . . . . . 22

4.2 The Energies for the Quantum Harmonic Oscillator. . . . . . . . . . . . . . . 23

4.3 The Aharonov-Bohm Effect $\ldots \ldots \ldots \ldots \ldots$. . . . . . . . . . . . . . . . 24

$\begin{array}{lll}5 \text { Conclusion } & 29\end{array}$

\begin{tabular}{|ll}
\hline A Derivation of Propagators & 30
\end{tabular}

A.1 The Wave-function in Terms of the Spatial Solutions of the Schrödinger Equation. 30

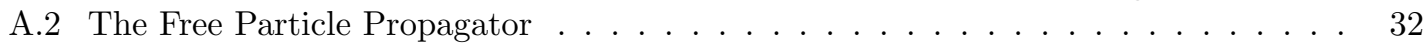

A.3 The Propagator for the Harmonic Oscillator . . . . . . . . . . . . . . . . . . . . . . 33

A.4 The Propagator for an Entangled Path with the Origin Removed . . . . . . . . . . 36 


\section{Introduction}

In the late $19^{\text {th }}$ century an inconsistency between statistical mechanics and electrodynamics was discovered that showed classical mechanics to be inadequate. The inconsistency between statistical mechanics and electrodynamics was clear to Einstein, Rayleigh and Jeans in 1905 when they realized independently that in thermodynamics all degrees of freedom of a system have an average energy of $\frac{1}{2} k T$, where $k$ is the Boltzmann constant and $T$ the environmental temperature. In electrodynamics the total number of frequency modes inside a cavity is inversely proportional to the square of the frequency, which followed the Rayleigh-Jeans law ${ }^{1}$

$$
B_{f}(T) \propto k T f^{2}
$$

where $B_{f}$ is the spectral radiance defined as the radiance through a surface per unit frequency. The higher the frequencies are, the spectral radiance associated with each frequency of the oscillators gets larger because of the dependence of the square of the frequency. It is an attempt to describe the spectral radiance of electromagnetic radiation at all wavelengths from a black body at a given temperature. Since all possible frequencies must be considered, the smaller the wavelength are the spectral radiance associated with each wavelength of the oscillators gets larger because the law is inversely proportional to the the fourth power of the wavelength $\lambda=c / f$. A contradiction existed in that when we sum over all the modes in the partition function ${ }^{2}$

$$
Z=\sum_{\bar{E}} e^{-\beta \bar{E}},
$$

with each average energy $\bar{E}$ equal to $k T$, the series does not converge. Max Planck resolved this by assuming that the energy of each harmonic oscillator came in a discrete form $E_{n}=n h f$, or quanta, where $n$ is a non-negative integer, $f$ is the frequency of the oscillator and $h$ a constant of proportionality now known as Planck's constant. Then if we sum over all integers $n$ the series converges to a finite number

$$
Z=\sum_{n}^{\infty} e^{-\beta n h f}=\frac{1}{e^{\beta h f}-1},
$$

called the Planck distribution. Later on the physicist Albert Einstein proposed an experiment that showed that when light at a certain frequency strikes a metal surface, electrons are released from it. The idea expressed in mathematical form is

$$
h f=E_{k}-E_{v},
$$

where $h f$ is the energy of a light quanta, $E_{k}$ the kinetic energy of the electrons and $E_{v}$ the energy involving the applied voltage for the electric field.

Another experiment, which shows that classical mechanics is insufficient in explaining phenomena, is the famous double-slit experiment. A source of particles are traveling freely to a wall where two separated slits have been carved out. If we designate one of the slits by A and the second by B, the particles have two options when they arrive to the wall: Either they can pass through slit A or slit B. At a distance after the slits we place detectors that counts how many particles that hits them. If we plot the number of detections with the position where the detector is positioned the shape of two bumps will be noticeable, where the height of them is the number of particles detected. Clearly the number of particles, at every position along the screen, vary each time we wish to perform the experiment. So all we can detect is the average number of particles hitting the screen after we have performed the experiment many times from equal initial conditions. If we move the experiment to a very small scale where the slits and the separation between them are very small our intuition tell us that nothing special will happen and the two bumps will again

\footnotetext{
${ }^{1}$ See David Bohm, Quantum Theory, p. 6

${ }^{2}$ See for instance David Chandler, Introduction to Modern Statistical Mechanics.
} 
be visible after performing the same experiments. However, this is not what we see because; when we again plot the total number of detections to position a wave-like pattern will appear in the distribution, just as what we expect when two water waves interfere with each other. This effect after scaling of an experiment is something that classical theories can not explain and a modification was in need. In the 1920's a new theory called Quantum Mechanics was developed and this theory agreed with experiment. Many formulations were developed; for example Werner Heisenberg's theory treats matrices using linear algebra and Erwin Schrödinger's theory deals with wave-functions commonly noted as $\psi$. The wave-function $\psi$ contains the description of the state of a system and the absolute square of $\psi$ is interpreted as the probability to find the system in a particular state. The wave-function satisfies the equation

$$
i \hbar \frac{\partial}{\partial t} \psi(x, t)=\hat{H} \psi(x, t)
$$

known as the Schrödinger equation, where $\hat{H}$ is the Hamiltonian operator and $\psi$ denotes the wave-function. 
In this thesis we are going to focus on another equivalent formulation of Quantum Mechanics that is based on the influential book of Quantum Mechanics by P.A.M. Dirad ${ }^{3}$ (and later developed by Richard P. Feynman 4 . Dirac assumed that a solution to the Schrödinger equation had the form

$$
\psi=A \exp \left\{i S_{c l} / \hbar\right\}=A \exp \left\{\frac{i}{\hbar} \int L_{c l} d t\right\},
$$

where $S_{c l}$ is the classical action. The classical action is found by using Lagrange's equations and substituting the resulting equation of motion back in the action and solve the time integral. This form of the wave-function can also be found in a paper from 1926 by Erwin Schrödinger $5^{5}$ were for the wave-function $\psi$ to have the statistical property it can be written as a product of wavefunctions corresponding to states occurring in succession. As the actions must have the additive property under the statistical property, we can find the relation between them as

$$
S=K \log \psi
$$

where $S$ is the classical action and $K$ is a constant with the dimensions of action. One easily sees that it can be re-written in the form of 1.0 .6 but with $K$ undetermined. If one substitutes this solution into the Schrödinger equation we obtain a differential equation for $S_{c l}$ and the normalization $A$. If we let $\hbar \rightarrow 0$ the resulting differential equation must reduce to the Hamilton-Jacobi equation from classical mechanics.

\section{Least Action in Classical Mechanics}

In this section we are going to look at how we can find the path $x(t)$ that minimizes the action. If we vary from the the path $x(t)$ a little bit then the action does not not change to a first order variation of the path and accordingly to the principle of least action we obtain the equation of motion. The action is a functional in the sense that it takes the whole form of the path $x(t)$ as input and outputs a number. For example, the action

$$
S[x(t)]=\int_{t_{1}}^{t_{2}} d t L(x(t), \dot{x}(t))
$$

is a functional of the whole path $x(t)$ where $L$ is the Lagrangian of the system. A functional can be viewed as a function of a function i.e. that depends on all the points of another function. To understand better what a functional is we may start with a function that depends only on certain points and then fill in more points so to smoothen out a line that can be drawn between the points. Say we have a function $F$ with a finite number of points $x\left(t_{1}\right), x\left(t_{2}\right), \cdots, x\left(t_{N}\right), \cdots$ :

$$
F=F\left(x\left(t_{1}\right), x\left(t_{2}\right), \cdots, x\left(t_{N}\right), \cdots\right) \text {. }
$$

The points $x\left(t_{1}\right), x\left(t_{2}\right), \cdots, x\left(t_{N}\right), \cdots$ represent different positions in time and together they represent the motion of the system. Say that we can connect the points by straight lines so that we have formed a polygonal curve, this curve is then an approximation to the motion of the system and the motion will be discontinuous at the joints of the curve. If we add more points to the function $F$ so that we can remove the discontinuities at the joints then the approximation of the motion gets more improved and we can draw a finer line between the points. We continue this process and add more discontinuities and hence if we formally pass the limit of number of points on the curve to infinity,

$$
\lim _{N \rightarrow \infty} F\left(x_{1}\left(t_{1}\right), x_{2}\left(t_{2}\right), \cdots, x\left(t_{N}\right), \cdots\right)=F[x(t)],
$$

\footnotetext{
${ }^{3}$ P.A.M Dirac, Quantum Mechanics, p. 121

${ }^{4}$ Richard P. Feynman, Principles of Least Action in Quantum Mechanics

${ }^{5}$ Annalen der Physik (4), vol. 79, 1926
} 
the curve gets smooth and we obtain what is known as a functional that depends on the form of the function $x(t)$. Now let us go back to the function $F$, which depends only on the points of the function $x(t)$, and suppose we vary the points by a small amount so that we can define a change in $F$ as

$$
\begin{aligned}
\Delta F & =F\left(x\left(t_{1}\right)+\sigma\left(t_{1}\right), x\left(t_{2}\right)+\sigma\left(t_{2}\right), \cdots, x\left(t_{N}\right)+\sigma\left(t_{N}\right), \cdots\right) \\
& -F\left(x\left(t_{1}\right), x\left(t_{2}\right), \cdots, x\left(t_{N}\right), \cdots\right)
\end{aligned}
$$

where $\sigma\left(t_{1}\right), \sigma\left(t_{2}\right), \cdots, \sigma\left(t_{N}\right), \cdots$ are small deviations from the points $x\left(t_{1}\right), x\left(t_{2}\right), \cdots, x\left(t_{N}\right), \cdots$. Then if we expand $\Delta F$ in a Taylor series expansion with a partial sum including only the $N$ first points of the function $F$ we get

$$
\begin{aligned}
\Delta F & =F\left(x\left(t_{1}\right)+\sigma\left(t_{1}\right), x\left(t_{2}\right)+\sigma\left(t_{2}\right), \cdots, x\left(t_{N}\right)+\sigma\left(t_{N}\right)\right)-F\left(x\left(t_{1}\right), x\left(t_{2}\right), \cdots, x\left(t_{N}\right)\right) \\
& =\sum_{i=1}^{N} \frac{\partial F}{\partial x\left(t_{i}\right)} \sigma\left(t_{i}\right)+\text { higher order terms }
\end{aligned}
$$

where we have omitted the trailing terms after the first order term for we are only interested in a lowest order variation of the functional $F$. If we let $N$ be taken in a limit such that the added points removes the discontinuities of the curve the change in the function $F$ becomes a change in the functional $F$ by an amount $\sigma(t)$, the sum becomes an integral and we obtain

$$
F[x(t)+\sigma(t)]=F[x(t)]+\int^{t} d \nu \frac{\delta F[x(\nu)]}{\delta x(\nu)} \sigma(\nu)+\cdots .
$$

The integrand term $\delta F[x(\nu)] / \delta x(\nu)$ is called a functional derivative and it is the functional derivative of $F$ with respect to the whole function $x(t)$.

If we replace $F$ by $S$ in 2.0 .13 and vary the path $x(t)$ by $\delta x(t)$ we get to a lowest order variation in $S$

$$
S[x(t)+\delta x(t)]=S[x(t)]+\int^{t} d \nu \frac{\delta S[x(\nu)]}{\delta x(\nu)} \delta x(\nu) .
$$

If we perform a variation of the action $S[x(\nu)]$ we obtain

$$
\begin{aligned}
\delta S[x(\nu)] & =S[x(\nu)+\delta x(\nu)]-S[x(\nu)] \\
& =\delta \int_{t_{a}}^{t_{b}} d \nu L(x(\nu), \dot{x}(\nu))=\int_{t_{a}}^{t_{b}} d \nu\left(\frac{\partial L}{\partial x(\nu)} \delta x(\nu)+\frac{\partial L}{\partial \dot{x}(\nu)} \delta \dot{x}(\nu)\right)
\end{aligned}
$$

where the motion of the system occur between two endpoints $x\left(t_{a}\right)$ and $x\left(t_{b}\right)$ and $\dot{x}(\nu)=d x(\nu) / d \nu$. The second term in the integrand contains a variation of the $\nu$-derivative and we can re-write this term as

$$
\delta \dot{x}(\nu)=\delta \lim _{\Delta \nu \rightarrow 0} \frac{x(\nu+\Delta \nu)-x(\nu)}{\Delta \nu}=\lim _{\Delta \nu \rightarrow 0} \frac{\delta x(\nu+\Delta \nu)-\delta x(\nu)}{\Delta \nu}=\frac{d}{d \nu} \delta x(\nu) .
$$

Then we can re-write the second term in 2.0 .15 by using the product-rule for derivatives as

$$
\frac{\partial L}{\partial \dot{x}(\nu)} \frac{d}{d \nu} \delta x(\nu)=\frac{d}{d \nu}\left(\frac{\partial L}{\partial \dot{x}(\nu)} \delta x(\nu)\right)-\delta x(\nu) \frac{d}{d \nu}\left(\frac{\partial L}{\partial \dot{x}(\nu)}\right) .
$$

If we substitute this in 2.0.15 we get

$$
\begin{aligned}
\delta S[x(\nu)] & =\int_{t_{a}}^{t_{b}} d \nu \frac{d}{d \nu}\left(\frac{\partial L}{\partial \dot{x}(\nu)} \delta x(\nu)\right)+\int_{t_{a}}^{t_{b}} d \nu \delta x(\nu)\left\{-\frac{d}{d \nu}\left(\frac{\partial L}{\partial \dot{x}(\nu)}\right)+\frac{\partial L}{\partial x(\nu)}\right\} \\
& =\left.\frac{\partial L}{\partial \dot{x}(\nu)} \delta x(\nu)\right|_{t_{a}} ^{t_{b}}+\int_{t_{a}}^{t_{b}} d \nu \delta x(\nu)\left\{-\frac{d}{d \nu}\left(\frac{\partial L}{\partial \dot{x}(\nu)}\right)+\frac{\partial L}{\partial x(\nu)}\right\} .
\end{aligned}
$$


Now if there are no variation at the endpoint $x\left(t_{a}\right)$ and $x\left(t_{b}\right)$ such that $\delta x\left(t_{a}\right)=\delta x\left(t_{b}\right)=0$, the first evaluation term vanishes and by comparing the variation of $S$ with the expression 2.0.13 we see that the functional derivative of $S$ with respect to $x(\nu)$ is

$$
\frac{\delta S[x(\nu)]}{\delta x(\nu)}=-\frac{d}{d \nu} \frac{\partial L}{\partial \dot{x}(\nu)}+\frac{\partial L}{\partial x(\nu)} .
$$

We wanted to find the path that makes the action functional an extremum and this occur when the action does not change to lowest order when we vary the path. This is the same as letting the functional derivative of the action $S$ with respect to $x(t)$ be zero and we obtain the equation of motion

$$
-\frac{d}{d \nu} \frac{\partial L}{\partial \dot{x}(\nu)}+\frac{\partial L}{\partial x(\nu)}=0
$$

This is known as the Euler-Lagrange equation and if we know the Lagrangian one finds an explicit expression of the equation of motion. Then one can solve for the path $x(t)$, which in this case is the classical path a particle always follow, and if we substitute the classical path denoted here by $x_{c l}(t)$ into the action $S$ one obtains the classical action $S_{c l}$.

\section{Least Action in Quantum Mechanics}

Now we wish to extend the least action principle to quantum mechanics.

\subsection{Summing over Paths}

Let us again consider the double-slit experiment described in the introduction. We want to explain mathematically why the distribution pattern in the double-slit experiment does not follow the classical ideas. Let us then consider this: Say we have both slits open, and a screen with detectors counting the total particles at each point along the screen. To each path a number $\phi\left[x_{j}\right]$ is assigned dependent on the whole form of $x_{j}$ where $x_{j}$ describes the classical path $j$ taken by the particle. Here we are assuming that the slit is small enough. Say that the particle takes the path through slit number 1 . Then the probability that a particle will take the path $x_{1}$ is

$$
P(\operatorname{path} 1)=\left|\phi\left[x_{1}\right]\right|^{2} .
$$

The number $P$ (path 1 ) should be interpreted as the probability to find the particle at the point $\left(x_{1}, t_{1}\right)$ given that it takes the path 1 . Likewise if the particle happens to follow the path 2 then the probability of finding the particle at the point $\left(x_{2}, t_{2}\right)$ is

$$
P(\text { path } 2)=\left|\phi\left[x_{2}\right]\right|^{2} \text {. }
$$

The classical treatment to get the probability that the particle selects any of the two paths 1 and 2 is simply obtained by adding the probabilities as

$$
P(\text { any path })=\left|\phi\left[x_{1}\right]\right|^{2}+\left|\phi\left[x_{2}\right]\right|^{2}
$$

since, for example, the probability of rolling either a three or a four on a die is the sum of the probabilities of rolling a three and a four respectively. If we collect all the measurements at the screen and plot the probability with position of each slit, with the other closed, we obtain two bell-shaped bumps residing next to each other with the peaks centered with the slit. Also we can clearly identify each bump with the respective slit that the particle entered so the particle can not end up around a point in the probability for slit 2 given it had entered slit 1 . The reason for that we have two bumps rather than two spikes centered in line with the slits is that when the particle enter, say, slit 1 there is a chance that its path will be refracted by the slit and be detected as a spread along the screen. Now as we mentioned earlier the wave-like distribution can not be explained by the probability 3.1 .3 since it does not reproduce a wave pattern. The 
solution to this is to treat each number $\phi\left[x_{j}\right]$ as a complex number and instead of absolute square each number $\phi\left[x_{j}\right]$ and add the probabilities we add the $\phi\left[x_{j}\right]$ 's then absolute square. What we obtain is something that is different from (3.1.3) that will contain additional terms. We have said that the $\phi\left[x_{j}\right]$ 's are complex numbers and we are going to call these numbers probability amplitudes and the absolute square of the probability amplitude will be called the probability. So the probability amplitude is the sum of all the alternatives that the particle can choose from and in the case of the double-slit experiment we have two alternatives: path 1 and path 2 . Then the total probability amplitude is

$$
K=\phi\left[x_{1}\right]+\phi\left[x_{2}\right]
$$

where we use the notation of the total probability amplitude as $K$ and we will keep this throughout this thesis. Then if we absolute square this and treat each probability amplitude as a complex number we get the probability of the particle to take either of the two paths as

$$
\begin{aligned}
P(\text { either path }) & =|K|^{2}=K^{*} K=\left(\phi\left[x_{1}\right]+\phi\left[x_{2}\right]\right)^{*}\left(\phi\left[x_{1}\right]+\phi\left[x_{2}\right]\right) \\
& =\left|\phi\left[x_{1}\right]\right|^{2}+\left|\phi\left[x_{2}\right]\right|^{2}+\phi^{*}\left[x_{2}\right] \phi\left[x_{1}\right]+\phi^{*}\left[x_{1}\right] \phi\left[x_{2}\right] .
\end{aligned}
$$

If we compare this with 3.1 .3 we see that these two probabilities is different from each other. It seems that there has appeared another term in the probability that can explain the wave-like pattern. Now, we can see that the first two terms remind us of the unperturbed problem when the particles can choose either slit and we will see two narrow bumps. We are going to postulate that the probability amplitudes have the form

$$
\phi\left[x_{j}(t)\right] \propto \exp \left\{i S\left[x_{j}(t)\right] / \hbar\right\},
$$

where $S\left[x_{j}\right]$ is the classical action for the path $x_{j}(t)$ and $\hbar=h / 2 \pi$ is the reduced Planck's constant. It is easy to extend the double-slit experiment to multiple slits. Then we have to sum up all probability amplitudes for each alternative that a particle can take and we can write a partial sum of the total amplitude

$$
K=\sum_{k=1}^{j} \phi\left[x_{k}\right] \propto \sum_{k=1}^{j} \exp \left\{i S\left[x_{k}\right] / \hbar\right\} .
$$

If we create even more holes in the wall of slits and add more walls with holes in them as well for the particles to pass through and if we pass the limit of amplitudes to infinity with the spacing between the paths to zero, as if there were infinitely many holes in the walls, and the amount of walls to infinity we can formally write 3.1 .7 as

$$
K=\int \mathcal{D} x(t) \exp \{i S[x(t)] / \hbar\}
$$

where $\mathcal{D} x(t)$ is a representation of the products of measures which corresponds to each wall, we sum over the paths for every amplitude as denoted by the integral symbol and the action $S[x(t)]$ is the classical action. $K$ is a function of the endpoints of the motion of the system known as the propagator and, as we will later see, can be used to find the probability of a state to make a transition into another state.

\subsection{Propagator with a Quadratic Lagrangian}

Now that we know the formal expression of the propagator we are going to show that if the Lagrangian is of a quadratic form in its position and velocity all we need is to find the classical action besides from a normalization constant depending only on the time at the endpoints where the motion occur. If we begin with the action

$$
S[x(t)]=\int_{t_{1}}^{t_{2}} d t L(\dot{x}(t), x(t)),
$$


that is an integral of the Lagrangian of a system going from a state at $\left(x\left(t_{1}\right), t_{1}\right)$ to a state at $\left(x\left(t_{2}\right), t_{2}\right)$, then we are using the principle of least action with the general quadratic Lagrangian

$$
L(x(t), \dot{x}(t))=a(t) x^{2}+b(t) x \dot{x}+c(t) \dot{x}^{2}+d(t) \dot{x}+e(t) x+f(t)
$$

and substitute the solutions of the equations of motion back into 3.2.1 to obtain the classical action. Now, if a particle follows a particular path $\bar{x}(t)$ where the dash denotes a classical path let us see what happens if we are moving along a path that is nearby the classical path. Let us call this path $x(t)$. Then we can write this path as a sum of the classical path $\bar{x}(t)$ and an arbitrary path $y(t)$ as

$$
x(t)=\bar{x}(t)+y(t)
$$

where $y(t)$ is zero at the endpoints. We can expand the left hand side of 3.2.2 in a Taylor series around $y(t)$ and $\dot{y}(t)$ as

$$
L(\dot{x}, x)=L(\dot{\bar{x}}, \bar{x})+\left.\frac{\partial L}{\partial x}\right|_{\bar{x}} y+\left.\frac{\partial L}{\partial \dot{x}}\right|_{\dot{\bar{x}}} \dot{y}+\left.\frac{1}{2}\left(\frac{\partial^{2} L}{\partial x^{2}} y^{2}+\frac{\partial^{2} L}{\partial x \partial \dot{x}} y \dot{y}+\frac{\partial^{2} L}{\partial \dot{x}^{2}} \dot{y}^{2}\right)\right|_{\bar{x}, \dot{\bar{x}}},
$$

where we have terminated the Taylor expansion to second order since it is assumed that the Lagrangian is at most quadratic. The first term on the right hand side is the Lagrangian itself and the trailing terms are functions of $y$ and $\dot{y}$, all evaluated on the classical path. Since the Lagrangian is at most quadratic in its terms the Taylor series terminate at second order in $y$ and $\dot{y}$. By using the chain rule on the third term on the right side of (3.2.4) we obtain

$$
\left.\frac{\partial L}{\partial \dot{x}}\right|_{\dot{\bar{x}}} \dot{y}=\frac{d}{d t}\left(\left.\frac{\partial L}{\partial \dot{x}}\right|_{\dot{\bar{x}}} y\right)-\left.\frac{d}{d t} \frac{\partial L}{\partial \dot{x}}\right|_{\dot{\bar{x}}} y .
$$

If we substitute this in (3.2.4) the second and third term becomes

$$
\begin{aligned}
\left.\frac{\partial L}{\partial x}\right|_{\bar{x}} y+\left.\frac{\partial L}{\partial \dot{x}}\right|_{\dot{\bar{x}}} \dot{y} & =\left.\frac{\partial L}{\partial x}\right|_{\bar{x}} y+\frac{d}{d t}\left(\left.\frac{\partial L}{\partial \dot{x}}\right|_{\dot{\bar{x}}} y\right)-\left.\frac{d}{d t} \frac{\partial L}{\partial \dot{x}}\right|_{\dot{\bar{x}}} y \\
& =\left(-\left.\frac{d}{d t} \frac{\partial L}{\partial \dot{x}}\right|_{\dot{\bar{x}}}+\left.\frac{\partial L}{\partial x}\right|_{\bar{x}}\right) y+\left.\frac{d}{d t} \frac{\partial L}{\partial \dot{x}}\right|_{\dot{\bar{x}}} y
\end{aligned}
$$

One can easily notice that the first term on the right hand side is the Euler-Lagrange equation which vanishes since the terms are evaluated at the classical path. By comparing the right hand side of 3.2.2 with the right side of 3.2.4 we can identify the partial derivatives after explicit calculation that

$$
\frac{\partial^{2} L}{\partial x^{2}}=2 a(t), \quad \frac{\partial^{2} L}{\partial x \partial \dot{x}}=b(t), \quad \frac{\partial^{2} L}{\partial \dot{x}^{2}}=2 c(t)
$$

Now by substituting the last term in 3.2 .6 and the explicit forms of the partial derivatives back in equation (3.2.4) we obtain

$$
L(x, \dot{x})=L(\bar{x}, \dot{\bar{x}})+\frac{d}{d t}\left(\left.\frac{\partial L}{\partial \dot{x}}\right|_{\dot{\bar{x}}} y\right)+\left.\frac{1}{2}\left(2 a(t) y^{2}+b(t) y \dot{y}+2 c(t) \dot{y}^{2}\right)\right|_{\bar{x}, \dot{\bar{x}}} .
$$

If we integrate both sides over the times $t_{1}$ and $t_{2}$ we get the action

$$
S[x(t)]=\int_{t_{1}}^{t_{2}} d t L(\bar{x}, \dot{\bar{x}})+\int_{t_{1}}^{t_{2}} d t \frac{d}{d t}\left(\left.\frac{\partial L}{\partial \dot{x}}\right|_{\dot{\bar{x}}} y\right)+\int_{t_{1}}^{t_{2}} d t \frac{1}{2}\left(2 a(t) y^{2}+b(t) y \dot{y}+2 c(t) \dot{y}^{2}\right) .
$$

One can see that on the right hand side there are three terms: The first term is the classical action, the second term an integral over a total time-derivative and it is a boundary term and the third term is a time integral over the path $y(t)$. Since the deviation $y(t)$ vanishes at the endpoints the second term vanish when evaluated there and we get

$$
S[x(t)]=S_{c l}+\int_{t_{1}}^{t_{2}} d t \frac{1}{2}\left(2 a(t) y^{2}+b(t) y \dot{y}+c(t) \dot{y}^{2}\right) .
$$


where $S_{c} l=S[\bar{x}(t)]$ is the classical action which is evaluated at the path that makes the action an extremum. We can see that the second term on the right hand side is a functional of the deviation $y$ and depends on the times at the endpoints and by substitution of the action in the formal path integral we get

$$
\begin{aligned}
& K\left(x_{2}, t_{2} ; x_{1}, t_{1}\right)= \\
& \int_{x\left(t_{1}\right)=x_{1}}^{x\left(t_{2}\right)=x_{2}} \mathcal{D} x(t) \exp \left\{\frac{i}{\hbar} S[\bar{x}(t)]+\frac{i}{\hbar} \int_{t_{1}}^{t_{2}} d t \frac{1}{2}\left(2 a(t) y^{2}+b(t) y \dot{y}+2 c(t) \dot{y}^{2}\right)\right\} .
\end{aligned}
$$

On the left hand side we have used the notation of a particle going from a point $\left(x_{1}, t_{1}\right)$ to a point $\left(x_{2}, t_{2}\right)$ as read from right to left. Under the path integration the first term on the right hand side in the integrand can be extracted outside the path integral since it does not depend on the deviation $y(t)$. What we have left is a path integral only dependent of the time at the endpoints and the classical path $\bar{x}(t)$ Let us denote the time-dependent path integral $A\left(t_{1}, t_{2}\right)$ then the propagator for the quadratic Lagrangian is

$$
\begin{aligned}
& K\left(x_{2}, t_{2} ; x_{1}, t_{1}\right)= \\
& \exp \{i S[\bar{x}(t)] / \hbar\} \int_{y\left(t_{1}\right)=0}^{y\left(t_{2}\right)=0} \mathcal{D} y(t) \exp \left\{\frac{i}{\hbar} \int_{t_{1}}^{t_{2}} d t \frac{1}{2}\left(2 a(t) y^{2}+b(t) y \dot{y}+2 c(t) \dot{y}^{2}\right)\right\} \\
& =A\left(t_{1}, t_{2}\right) \exp \{i S[\bar{x}(t)] / \hbar\} .
\end{aligned}
$$

This is a really interesting result. We sought an extension of the classical mechanics to quantum mechanics by first assigning a number to each amplitude that a particle can possibly take. Then by postulating the form of the amplitudes we constructed the propagator that can be used to find the relation between states of a system. Since it is impossible to know which path a particle follows we can only talk about the probability that a particle reaches a certain state given that it has been in a previous state. When the majority of paths resides near the classical path $\bar{x}(t)$ then if the Lagrangian is of quadratic form it suffice only to calculate the classical action of the path $\bar{x}(t)$, besides from a normalization constant $A\left(t_{1}, t_{2}\right)$, to find the explicit expression of the propagator. We shall later see how the propagator can be applied in problems.

\subsection{Separating the Propagator into Multiple Propagations}

There exists situations where the motion of a system is separated in many propagations for example a particle might move from a state at $\left(x_{a}, t_{a}\right)$ to a state at $\left(x_{c}, t_{c}\right)$ and then later on from a state at $\left(x_{c}, t_{c}\right)$ to a state at $\left(x_{b}, t_{b}\right)$. First let us consider the propagation from a point $\left(x_{a}, t_{a}\right)$ to a point $\left(x_{b}, t_{b}\right)$ then the propagator is

$$
K\left(x_{b}, t_{b} ; x_{a}, t_{a}\right)=\int_{x\left(t_{a}\right)=x_{a}}^{x\left(t_{b}\right)=x_{b}} \mathcal{D} x(t) \exp \left\{\frac{i}{\hbar} \int_{t_{a}}^{t_{b}} d t L(x, \dot{x}, t)\right\}
$$

where we have included a possible explicit time dependence in the Lagrangian for generality. If we now separate the motion of the particle as mentioned in the beginning of the section then the integrand of the propagator can be written as

$$
\exp \left\{i S_{a b} / \hbar\right\}=\exp \left\{i\left[S_{a c}+S_{c b}\right] / \hbar\right\}
$$

where the notation $S_{a b}$ says that we are integrating the Lagrangian from the time $t_{a}$ to the time $t_{b}$. First we look at the motion from $\left(x\left(t_{a}\right), t_{a}\right)$ to $\left(x\left(t_{c}\right), t_{c}\right)$. To find the propagator we integrate (3.3.2) over all paths from $\left(x\left(t_{a}\right), t_{a}\right)$ to $\left(x\left(t_{c}\right), t_{c}\right)$. The action from the point $\left(x\left(t_{c}\right), t_{c}\right)$ to point $\left(x\left(t_{b}\right), t_{b}\right)$ remains stationary and can be extracted to the outside of the path integral hence we 
obtain the expression

$$
\begin{aligned}
& \exp \left\{i S_{c b} / \hbar\right\} \int_{x\left(t_{a}\right)=x_{a}}^{x\left(t_{c}\right)=x_{c}} D x(t) \exp \left\{i S_{a c} / \hbar\right\} \\
= & \exp \left\{i S_{c b} / \hbar\right\} K\left(x_{c}, t_{c} ; x_{a}, t_{a}\right) .
\end{aligned}
$$

If we Integrate 3.3 .3 over all paths between the points $\left(x_{c}, t_{c}\right)$ and $\left(x_{b}, t_{b}\right)$ we obtain

$$
\int_{x\left(t_{c}\right)=x_{c}}^{x\left(t_{b}\right)=x_{b}} \exp \left\{i S_{c b} / \hbar\right\} K\left(x_{c}, t_{c} ; x_{a}, t_{a}\right) .
$$

What we have obtained is however not the same as 3.3 .1 since the propagation is free between the endpoints and the way we have constructed the propagator in separating the motion in two steps restricts the motion such that when the system moves from $\left(x_{a}, t_{a}\right)$ to $\left(x_{b}, t_{b}\right)$ it will only pass through the point $\left(x_{c}, t_{c}\right)$. So in order to have this construction of the propagator to agree with 3.3 .1 we must integrate over all points $x\left(t_{c}\right)=x_{c}$ :

$$
\begin{aligned}
K\left(x_{b}, t_{b} ; x_{a}, t_{a}\right) & = \\
& =\int_{-\infty}^{\infty} d x_{c} K\left(x_{c}, t_{c} ; x_{a}, t_{a}\right) \int_{x\left(t_{c}\right)=x_{c}}^{x\left(t_{b}\right)=x_{b}} D x(t) \exp \left\{i S_{c b} / \hbar\right\} \\
& =\int_{-\infty}^{\infty} d x_{c} K\left(x_{b}, t_{b} ; x_{c}, t_{c}\right) K\left(x_{c}, t_{c} ; x_{a}, t_{a}\right) .
\end{aligned}
$$

One can realize that if we have more than two events the propagation between two points can easily be extended to multiple propagations: If we let the system propagate between $\left(x_{b}, t_{b}\right)$ and a point, say, $\left(x_{d}, t_{d}\right)$ then we can multiply both sides of 3.3 .5 by $K\left(x_{d}, t_{d} ; x_{b}, t_{b}\right)$ and integrate over $\left(x_{b}, t_{b}\right)$ to obtain

$$
\begin{aligned}
K\left(x_{d}, t_{d} ; x_{a}, t_{a}\right) & =\int_{-\infty}^{\infty} d x_{b} K\left(x_{d}, t_{d} ; x_{b}, t_{b}\right) K\left(x_{b}, t_{b} ; x_{a}, t_{a}\right) \\
& =\int_{-\infty}^{\infty} \int_{-\infty}^{\infty} d x_{b} d x_{c} K\left(x_{d}, t_{d} ; x_{b}, t_{b}\right) K\left(x_{b}, t_{b} ; x_{c}, t_{c}\right) K\left(x_{c}, t_{c} ; x_{a}, t_{a}\right) .
\end{aligned}
$$

The process is easily iterated for any number of times and we can write the general result for $N$ consecutive propagations with $N-1$ separations between them as

$$
K\left(x_{N}, t_{N} ; x_{0}, t_{0}\right)=\int_{-\infty}^{\infty} \int_{-\infty}^{\infty} \cdots \int_{-\infty}^{\infty} \prod_{j=1}^{N-1} d x_{j} \prod_{j=1}^{N} K\left(x_{j}, t_{j} ; x_{j-1}, t_{j-1}\right)
$$

which is the propagator for a system to move between the point $x\left(t_{0}\right)=x_{0}$ and the point $x\left(t_{N}\right)=$ $x_{N}$ where $x_{0}=x_{a}$ and $x_{N}=x_{b}$. In the next subsection we are going to investigate the expression (3.3.9) further.

\subsection{Writing the full Propagator with a Time-Slicing Method}

From the previous result 3.3 .9 we can explicitly calculate the formal path integral 3.1 .8 by a method known as time-slicing. The idea is to break up a propagation into multiple parts where each propagation is separated equally in time where the time is a small quantity and then take a limiting procedure of the time separation. Let us define the time separation between the separate paths as $\epsilon=\left(t_{b}-t_{a}\right) / N$ where $t_{a}$ and $t_{b}$ are the times at the endpoints and $N$ the number of 


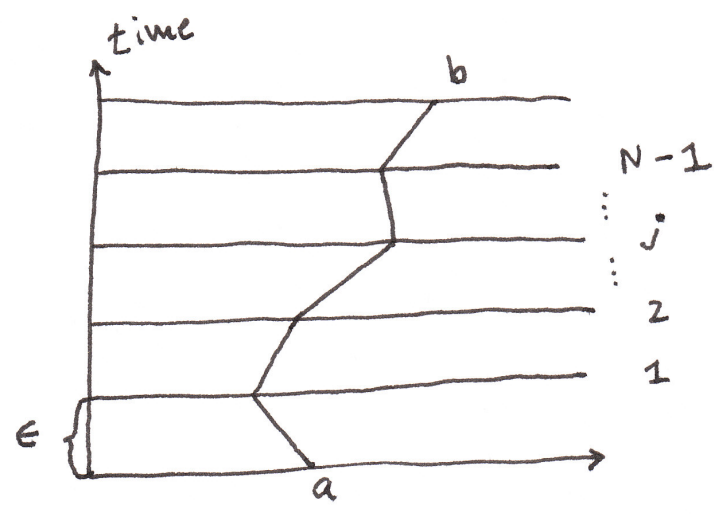

Figure 1: The path, from a point $a$ to a point $b$, can be separated into multiple paths separated equally in a time $\epsilon$. To the right at each line the numbers $1,2, j$ and $N-1$, denote the different points where the total path between $a$ and $b$ has been separated. The dots to the right indicates that there are more spacings in time in between.

separated paths. If $\epsilon$ is small we can approximate the propagator $K\left(x_{j-1}, t_{j-1} ; x_{j}, t_{j}\right)$ in 3.3 .9 as

$$
K\left(x_{j-1}, t_{j-1} ; x_{j}, t_{j}\right) \approx \frac{1}{A} \exp \{i \epsilon L / \hbar\},
$$

where we have included a normalization constant $A$, which is dependent on $\epsilon$, such that the propagator tends to a delta distribution, $\delta\left(x_{j-1}-x_{j}\right)$, when $\epsilon \rightarrow 0$. The Lagrangian must be written in a discretized form such that when we let $\epsilon \rightarrow 0$ or $N \rightarrow \infty$ the propagator coincides with the form (3.3.1). In Richard Feynman's PhD thesi: 6 the use of the following approximation for the Lagrangian

$$
L=L\left(\frac{x_{j}-x_{j-1}}{\epsilon}, \frac{x_{j}+x_{j-1}}{2}, \frac{t_{j}+t_{j-1}}{2}\right)
$$

is made and we have included a possible explicit appearance of time in the Lagrangian. The first term is the average velocity, the second term is the average position and the third term is the average time between two successive states. If we use the expression 3.4.2) in (3.4.1), substitute the result in 3.3 .9 and take the limit of $\epsilon \rightarrow 0$ we will obtain the exact expression of the propagator as

$$
\begin{aligned}
& K\left(x_{b}, t_{b} ; x_{a}, t_{a}\right)= \\
& \lim _{\epsilon \rightarrow 0} A^{-(N-1)} \int \cdots \int \prod_{j=1}^{N-1} d x_{j} \prod_{j=1}^{N} \exp \left\{\frac{i}{\hbar} \epsilon L\left(\frac{x_{j}-x_{j-1}}{\epsilon}, \frac{x_{j}+x_{j-1}}{2}, \frac{t_{j}+t_{j-1}}{2}\right)\right\}
\end{aligned}
$$

where it is known that $\left(x_{0}, t_{0}\right)=\left(x_{a}, t_{a}\right)$ and $\left(x_{N}, t_{N}\right)=\left(x_{b}, t_{b}\right)$. This formula can always be used to calculate the propagator explicitly but if the Lagrangian is of a quadratic form 3.2 .2 then it might save time to use 3.2 .13 .

\subsection{Orthonormality Condition between Propagators}

So far we have found several ways of writing the formal expression of the propagator and now we are looking for a orthonormality condition between different propagators. As we have mentioned before the propagator for a system being in a position $x\left(t_{b}\right)$ at a time $t_{b}$ given that it was at a position $x\left(t_{a}\right)$ at a time $t_{a}$ is written as $K\left(x_{b}, t_{b} ; x_{a}, t_{a}\right)$ where we are reading this expression from right to left. The wave-function is in some sense more general than the propagator, since the wave-function can have any initial conditions while the propagator is always a delta function at some particular point in time. If we do not know the initial conditions, or maybe not even

${ }^{6}$ Principle of least action in quantum mechanics, p. 31 
interested in them, we can change to the notation

$$
K\left(x, t ; x^{\prime}, t^{\prime}\right) \rightarrow \psi(x, t)
$$

and if we apply this to equation 3.3.5 we get in turn the relation

$$
\psi(x, t)=\int_{-\infty}^{\infty} d x^{\prime} K\left(x, t ; x^{\prime}, t^{\prime}\right) \psi\left(x^{\prime}, t^{\prime}\right) .
$$

Thus the relation between a previous state at $\left(x^{\prime}, t^{\prime}\right)$ and a present state at $(x, t)$ is related by the propagator $K\left(x, t ; x^{\prime}, t^{\prime}\right)$. In section 3.1 we postulated that the probability density of finding a state with certain coordinates given that it was in a previous state should be proportional to the absolute square of the propagator $K$. Then the probability of finding a system in a position $x$ at a time $t$, with no knowledge of initial conditions, must be

$$
P(x, t) \propto|\psi(x, t)|^{2} .
$$

The probability of finding the system in any state given a time $t$ must be

$$
1=\int_{-\infty}^{\infty} d x P(x, t)=\int_{-\infty}^{\infty} d x|\psi(x, t)|^{2} .
$$

By substituting 3.5.2 into 3.5.4 we obtain

$$
\begin{aligned}
& \int_{-\infty}^{\infty} d x|\psi(x, t)|^{2} \\
& =\int_{-\infty}^{\infty} d x \int_{-\infty}^{\infty} d y K^{*}(x, t ; y, T) \psi^{*}(y, T) \int_{-\infty}^{\infty} d y^{\prime} K\left(x, t ; y^{\prime}, T\right) \psi\left(y^{\prime}, T\right) \\
& =\int_{-\infty}^{\infty} \int_{-\infty}^{\infty} \int_{-\infty}^{\infty} d x d y d y^{\prime} K^{*}(x, t ; y, T) K\left(x, t ; y^{\prime}, T\right) \psi^{*}(y, T) \psi\left(y^{\prime}, T\right) .
\end{aligned}
$$

Here $\psi^{*}(x, t)$ is the complex conjugate of the wave-function. Since we have said that 3.5 .4 is independent of the time it means that the expression on the right hand side of (3.5.4) stay the same however we change the time:

$$
\int_{-\infty}^{\infty} d x|\psi(x, t)|^{2}=\int_{-\infty}^{\infty} d y\left|\psi\left(y, t^{\prime}\right)\right|^{2} .
$$

Now we must get (3.5.8) from 3.5.5) and this can be found by using the following trick: If we use the definition of the Dirac delta function we can write the right hand side of $(3.5 .8)$ as

$$
\int d y|\psi(y, t)|^{2}=\int_{-\infty}^{\infty} \int_{-\infty}^{\infty} d y d y^{\prime} \delta\left(y-y^{\prime}\right) \psi^{*}\left(y, t^{\prime}\right) \psi\left(y^{\prime}, t^{\prime}\right)
$$

where we have used the fact that the probability of finding a system in a state is invariant if one changes the time and by comparing this result with 3.5.5 we must have the relation

$$
\int_{-\infty}^{\infty} d x K^{*}\left(x, t ; y, t^{\prime}\right) K\left(x, t ; y^{\prime}, t^{\prime}\right)=\delta\left(y-y^{\prime}\right) .
$$

So if we know the propagator it must obey this relation. 


\subsection{The Relation of the Path Integral to the Schrödinger Equation}

The equivalence of the path integral formulation to the Schrödinger formulation will soon be evident when we are considering a small time interval and Taylor expand around it. The kinetic part of the Lagrangian is assumed to be quadratic in the velocities and the potential to be dependent of position and, for generality, the time. The described Lagrangian is

$$
L=\frac{1}{2} m \dot{x}^{2}-V(x, t) .
$$

From the result of equation 3.5 .2 we get

$$
\psi(x, t+\epsilon)=\int_{-\infty}^{\infty} d y K(x, t+\epsilon ; y, t) \psi(y, t) .
$$

where the distance between $y$ and $x$ must be small enough to be valid for non-relativistic considerations and $\epsilon$ is the time of propagation. If we assume that $\epsilon$ is small we can approximate the propagator by using $(3.4 .2$ as

$$
\begin{aligned}
K(x, t+\epsilon ; y, t) & =\frac{1}{A} \exp \left\{\frac{i}{\hbar} \epsilon L\left(\frac{x-y}{\epsilon}, \frac{x+y}{2}, \frac{2 t+\epsilon}{2}\right)\right\} \\
& =\frac{1}{A} \exp \left\{\frac{i}{\hbar}\left[\frac{m(x-y)^{2}}{2 \epsilon}-\epsilon V\left(\frac{x+y}{2}, t+\frac{\epsilon}{2}\right)\right]\right\}
\end{aligned}
$$

where $1 / A$ is a normalization constant determined later on. Replacing (3.6.3) in (3.6.2) and changing variables to $x=y-\eta$ gives

$$
\psi(x, t+\epsilon)=\frac{1}{A} \int_{-\infty}^{\infty} d \eta \exp \left\{\frac{i}{\hbar}\left[\frac{m \eta^{2}}{2 \epsilon}-\epsilon V\left(x+\frac{\eta}{2}, t+\frac{\epsilon}{2}\right)\right]\right\} \psi(x+\eta, t)
$$

Since $\epsilon V$ is small to first order in $\epsilon$ we can write

$$
\begin{aligned}
\epsilon V\left(x+\frac{\eta}{2}, t+\frac{\epsilon}{2}\right) & \approx \epsilon V(x, t)+\epsilon \frac{\eta}{2} \frac{\partial V}{\partial x}+\frac{\epsilon^{2}}{2} \frac{\partial V}{\partial t} \\
& \approx \epsilon V(x, t) .
\end{aligned}
$$

where $\eta$ is small. Hence we can rewrite 3.6 .4 as

$$
\psi(x, t+\epsilon)=\frac{1}{A} \int_{-\infty}^{\infty} d \eta \exp \left\{\frac{i}{\hbar}\left[\frac{m \eta^{2}}{2 \epsilon}-\epsilon V(x, t)\right]\right\} \psi(x+\eta, t) .
$$

Since $\epsilon$ and $\eta$ are small quantities we can expand both sides in a Taylor series as

$$
\begin{gathered}
\psi(x, t)+\epsilon \frac{\partial \psi}{\partial t}+\cdots= \\
\frac{1}{A} \int_{-\infty}^{\infty} d \eta\left\{\exp \left(\frac{i m \eta^{2}}{2 \hbar \epsilon}\right)[1-(i / \hbar) \epsilon V(x, t)+\cdots]\left(\psi(x, t)+\eta \frac{\partial \psi}{\partial x}+\frac{\eta^{2}}{2 !} \frac{\partial^{2} \psi}{\partial x^{2}} \cdots\right)\right\}
\end{gathered}
$$

The expansion in the potential and the left hand side of (3.6.7) can be terminated after first order in $\epsilon$ and we see that we must keep at least second order in $\eta$ since all odd orders in $\eta$ cancels out under Gaussian integration. If we neglect theses terms 3.6.7 becomes instead

$$
\psi(x, t)+\epsilon \frac{\partial \psi}{\partial t}=\frac{1}{A}[1-(i / \hbar) \epsilon V(x, t)] \int_{-\infty}^{\infty} d \eta \exp \left\{\frac{i m \eta^{2}}{2 \hbar \epsilon}\right\}\left\{\psi(x, t)+\frac{\eta^{2}}{2 !} \frac{\partial^{2} \psi}{\partial x^{2}}\right\}
$$

The wave-function and its second derivative in position can be factored out from the Gaussian integral since they do not depend on $\eta$. The first integral on the right hand side is

$$
\int_{-\infty}^{\infty} d \eta \exp \left\{\frac{i m \eta^{2}}{2 \hbar \epsilon}\right\}=\sqrt{\frac{2 \pi i \hbar \epsilon}{m}}
$$


The second integral can be written as

$$
\begin{aligned}
& \int_{-\infty}^{\infty} d \eta \frac{\eta^{2}}{2} \exp \left\{\frac{i m \eta^{2}}{2 \hbar \epsilon}\right\}=\frac{\partial}{\partial(i m / \hbar \epsilon)} \int_{-\infty}^{\infty} d \eta \exp \left\{\frac{i m \eta^{2}}{2 \hbar \epsilon}\right\} \\
= & \frac{\partial}{\partial(i m / \hbar \epsilon)}\left(\frac{2 \pi i \hbar \epsilon}{m}\right)^{1 / 2}=-\frac{1}{2}\left(\frac{-i m}{2 \pi \hbar \epsilon}\right)^{-3 / 2}\left(-\frac{1}{2 \pi}\right)=\frac{1}{4 \pi}\left(\frac{2 \pi i \hbar \epsilon}{m}\right)^{3 / 2} .
\end{aligned}
$$

where we have used differentiation under the integral sign. If we substitute the result for the integrals in 3.6 .8 we get

$$
\psi(x, t)+\epsilon \frac{\partial \psi}{\partial t}=\frac{1}{A}[1-(i / \hbar) \epsilon V(x, t)]\left\{\psi(x, t) \sqrt{\frac{2 \pi i \hbar \epsilon}{m}}+\frac{\partial^{2} \psi}{\partial x^{2}} \frac{1}{4 \pi}\left(\frac{2 \pi i \hbar \epsilon}{m}\right)^{3 / 2}\right\}
$$

We see that if we compare both sides we can identify

$$
\psi(x, t)=\psi(x, t) \frac{1}{A} \sqrt{\frac{2 \pi i \hbar \epsilon}{m}}
$$

so the normalization constant $A$ is

$$
A=\sqrt{\frac{2 \pi i \hbar \epsilon}{m}}
$$

Then after replacing $A$ in 3.6 .8 we obtain

$$
\begin{aligned}
\psi(x, t)+\epsilon \frac{\partial \psi}{\partial t} & =[1-(i / \hbar) \epsilon V(x, t)]\left\{\psi(x, t)+\frac{i \hbar \epsilon}{2 m} \frac{\partial^{2} \psi}{\partial x^{2}}\right\} \\
& =\psi(x, t)+\frac{i \hbar \epsilon}{2 m} \frac{\partial^{2} \psi}{\partial x^{2}}-\frac{i}{\hbar} \epsilon V(x, t) \psi(x, t)-\frac{\epsilon^{2}}{2 m} \frac{\partial^{2} \psi}{\partial x^{2}} V(x, t) .
\end{aligned}
$$

By transposing terms, neglecting higher order in $\epsilon$, multiplying both sides by $1 / \epsilon$ and let $\epsilon \rightarrow 0$ we obtain in this limit the exact equation

$$
i \hbar \frac{\partial}{\partial t} \psi(x, t)=-\frac{\hbar^{2}}{2 m} \frac{\partial^{2}}{\partial x^{2}} \psi(x, t)+V(x, t) \psi(x, t) .
$$

This is of course the one-dimensional time-dependent Schrödinger equation.

\subsection{Obtaining the Hamilton-Jacobi Equation from a Wave Function}

One of the fundamentals in quantum mechanics is that when we let $\hbar \rightarrow 0$ we must go over into classical mechanics. The wave-function can be written in terms of an exponential being proportional to the classical action

$$
\psi=A \exp \left(i S_{c l} / \hbar\right)
$$

where $\psi=\psi(x, t)$ and $A$ is some time-independent constant. Thus by substituting this solution in the Schrödinger equation we get

$$
-\psi \frac{\partial S_{c l}}{\partial t}=-\psi \frac{i \hbar}{2 m}\left\{\frac{\partial^{2} S_{c l}}{\partial x^{2}}+\frac{i}{\hbar}\left(\frac{\partial S_{c l}}{\partial x}\right)^{2}\right\}+\psi V .
$$

By dropping the wave-function $\psi$ on both sides and letting $\hbar \rightarrow 0$ we should go over into classical mechanics. If we do so we obtain

$$
-\frac{\partial S_{c l}}{\partial t}=\frac{1}{2 m}\left(\frac{\partial S_{c l}}{\partial x}\right)^{2}+V
$$

which indeed is a Hamilton-Jacobi equation from classical mechanics. 


\subsection{The Propagator in terms of Solutions of the Schrödinger Equation}

The propagator can also be represented as a discrete sum over the solutions of the Schrödinger equation as shown in the appendix. If we substitute

$$
c_{n}=a_{n} \exp \left(i E_{n} t^{\prime} / \hbar\right)=\exp \left(i E_{n} t^{\prime} / \hbar\right) \int_{-\infty}^{\infty} d x X_{n}^{*}(x) \psi\left(x, t^{\prime}\right)
$$

in $A .1 .10$ we obtain the result

$$
\begin{aligned}
\psi(x, t) & =\sum_{n} c_{n} X_{n}(x) \exp \left(-i E_{n} t / \hbar\right) \\
& =\sum_{n}\left[\exp \left(i E_{n} t^{\prime} / \hbar\right) \int_{-\infty}^{\infty} d x^{\prime} X_{n}^{*}\left(x^{\prime}\right) \psi\left(x^{\prime}, t^{\prime}\right)\right] X_{n}(x) \exp \left(-i E_{n} t / \hbar\right) \\
& =\int_{-\infty}^{\infty} d x^{\prime} \sum_{n} X_{n}^{*}\left(x^{\prime}\right) X_{n}(x) \exp \left(-i E_{n}\left(t-t^{\prime}\right) / \hbar\right) \psi\left(x^{\prime}, t^{\prime}\right) .
\end{aligned}
$$

Then by comparing this with $(3.5 .2)$ we finally arrive at

$$
K\left(x, t ; x^{\prime}, t^{\prime}\right)=\sum_{n} X_{n}(x) X_{n}^{*}\left(x^{\prime}\right) \exp \left(i E_{n}\left(t-t^{\prime}\right) / \hbar\right), \quad t>t^{\prime}
$$

This expression of the path integral in terms of the solutions to the Schrödinger equation can for example be applied in the extraction of the energy levels of the harmonic oscillator as will be shown in a later section.

\section{Applications of the Path Integral}

So far we have found some useful properties of the propagator. With the help of the results obtained from earlier investigations we are going to apply the properties of the propagator to some problems. For the interested the explicit calculations of the free, harmonic and path-constraint propagator can be found in the appendix.

\subsection{Slits}

Now we are going to apply the free particle propagator 7

$$
K\left(x, t ; x^{\prime}, t^{\prime}\right)=\sqrt{\frac{m}{2 \pi i \hbar\left(t-t^{\prime}\right)}} \exp \left\{\frac{i m}{2 \hbar} \frac{\left(x-x^{\prime}\right)^{2}}{t-t^{\prime}}\right\}
$$

to three slit problems: The single square slit, the single Gaussian slit and the double Gaussian slit. One can easily generalize the results to higher spatial dimensions since the propagator of higher spatial dimensions is simply the product of propagators for each single dimension. We will see that the physics, that can be obtained from the square slit, gets obscured by relatively complicated mathematics and we will introduce a Gaussian function as to ease the analysis and direct us faster to the physical results.

\subsubsection{Single Slit of a Square Type}

Imagine a freely moving particle traveling from a point A to another point B in a given time. It has no restrictions in its movement but given its departure from A it must end up at B. Now, say we obstruct the particles movement by inserting a wall with a small hole in it. The particle is still moving between point $\mathrm{A}$ and $\mathrm{B}$ but it is not moving freely, it must pass the hole in the wall to

\footnotetext{
${ }^{7}$ See the appendix for derivation.
} 
complete its travel between A and B. How can we translate this idea to the use of propagators? The basic idea is to use the successive propagation rule 3.3 .5 to find the state of a system given that it has been restricted in its free propagation. Say we have a particle in an initial state at an origo $(0,0)$ then we know with complete certainty that the particle was in that state every time we wish to restart the experiment. Let us start with constructing a free particle propagation using the result of 3.3 .5 . The motion of a particle from an origo $(0,0)$ to a point $(X+y, T)$ and a successive propagation to the point $\left(X+x^{\prime}, T+t^{\prime}\right)$ given that it was in the position $(X+y, T)$ is written as the product of the two propagators, $K\left(X+x^{\prime}, T+t^{\prime} ; X+y, T\right) K(X+y ; 0,0)$, where $X$ is the distance from the point of departure to the slit and $y$ is any point along the slit opening (see figure 2). Now, to obtain the amplitude to be at the point $X+x^{\prime}$ at a time $T+t^{\prime}$, we must integrate over all possible positions $y$. Hence we obtain

$$
K\left(X+x^{\prime}, T+t^{\prime} ; 0,0\right)=\int_{-\infty}^{\infty} d y K\left(X+x^{\prime}, T+t^{\prime} ; X+y, T\right) K(X+y, T ; 0,0)
$$

where the position $X$ is some point residing along the separation $y$. We are only interested in the particle which enter through the slit, and by neglecting any particles that do not enter the slit we can change the integration limits so to exclude the contributing amplitudes for the particles not entering the slit. So let us restrict the propagation to a gap of width $2 b$ where $b$ is some constant. Since the integration interval is now $(-b, b)$ instead of $(-\infty, \infty)$ we have only allowed the particles passing through the slit and neglected those who do not enter.

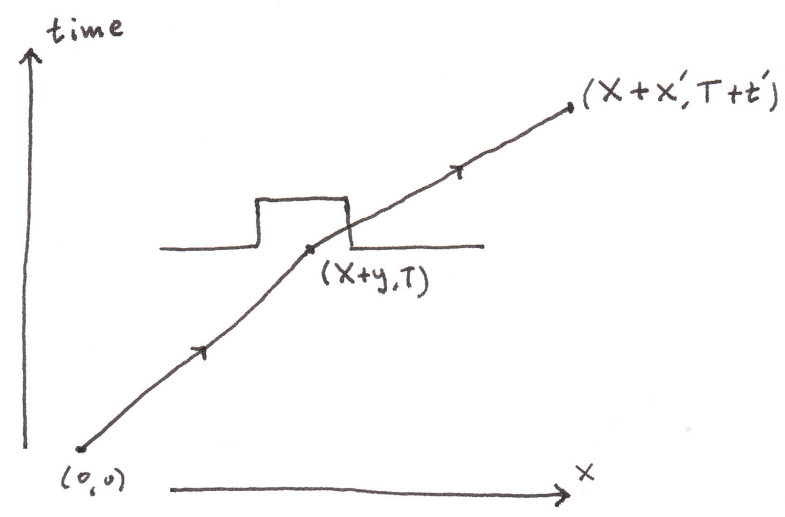

Figure 2: A formal view of the single square slit setup where time is upward and position is to the right in the figure. The particle travels freely from a point $(0,0)$ to the slit at the point $(X+y, T)$. If the particle is passing though the slit it contributes to the amplitude and travels further to a point $X+x^{\prime}, T+t^{\prime}$. As it is uncertain to where in the slit the particle might have passed through, we must integrate over the width over slit to obtain the total amplitude. Any particle that do not pass through the slit does not contribute to the total amplitude.

The propagator when summing over all amplitudes between $-b$ and $b$ becomes

$$
K\left(X+x^{\prime}, T+t^{\prime} ; 0,0\right)=\int_{-b}^{b} d y K\left(X+x^{\prime}, T+t^{\prime} ; X+y, T\right) K(X+y, T ; 0,0) .
$$

The particle is released from the origo at a time $t=0$ and travels to the point $X+y$ at a time $T$, where it must be found in a position interval $(X-b, X+b)$ since otherwise we have no contribution to the amplitude. If it is detected in this interval, it contributes to the amplitude and travels further to the point $X+x^{\prime}$ at a time $T+t^{\prime}$. Then we sum over all possible propagations within the interval $-b$ and $b$, since it uncertain to where in the position interval the particle might be. Since the propagation is free we can replace the propagators on the right hand side with the free particle propagator, A.2.10, and obtain an expression of the propagation as

$$
\begin{aligned}
K\left(x^{\prime}+X, t^{\prime}+T ; 0,0\right) & =\int_{-b}^{b} d y K\left(x^{\prime}+X, t^{\prime}+T ; X+y, T\right) K(X+y, T ; 0,0) \\
& =C \frac{m}{2 \pi i \hbar \sqrt{t^{\prime} T}} \int_{-b}^{b} d y \exp \left\{\frac{i m}{2 \hbar}\left[\frac{(X+y)^{2}}{T}+\frac{\left(x^{\prime}-y\right)^{2}}{t^{\prime}}\right]\right\}
\end{aligned}
$$


where we have included a constant $C$ to be determined by normalization. Next, we complete the square in the integral to obtain

$$
\begin{aligned}
& K\left(x^{\prime}+X, t^{\prime}+T ; 0,0\right)= \\
& C \frac{m}{2 \pi i \hbar \sqrt{t^{\prime} T}} \exp \left\{\frac{i m}{2 \hbar}\left[\frac{X^{2}}{T}+\frac{x^{\prime 2}}{t^{\prime}}\right]\right\} \exp \left\{\frac{i m}{2 \hbar t^{\prime}}\left(1+\frac{t^{\prime}}{T}\right)^{-1}\left(x^{\prime}-V t^{\prime}\right)^{2}\right\} \\
& \times \int_{-b}^{b} d y \exp \left\{\frac{i m}{2 \hbar t^{\prime}}\left(1+\frac{t^{\prime}}{T}\right)^{-1}\left(y\left(1+\frac{t^{\prime}}{T}\right)-\left(x^{\prime}-V t^{\prime}\right)\right)^{2}\right\}
\end{aligned}
$$

and by a change of variable

$$
i w=\sqrt{\frac{i m}{2 \hbar t^{\prime}}} \frac{\left(x^{\prime}-V t^{\prime}\right)-y\left(1+t^{\prime} / T\right)}{\sqrt{1+t^{\prime} / T}}
$$

we can rewrite the propagator as

$$
\begin{aligned}
& K\left(x^{\prime}+X, t^{\prime}+T ; 0,0\right)= \\
& C \sqrt{\frac{m}{\pi^{2} i \hbar T}}\left(1+\frac{t^{\prime}}{T}\right)^{-1 / 2} \exp \left\{\frac{i m}{2 \hbar}\left[\frac{X^{2}}{T}+\frac{x^{\prime 2}}{t^{\prime}}+\frac{1}{t^{\prime}}\left(x^{\prime}-V t^{\prime}\right)\left(1+\frac{t^{\prime}}{T}\right)^{-1}\right]\right\} \int_{\eta_{-} / i}^{\eta_{+} / i} d w e^{-w^{2}}
\end{aligned}
$$

where we have defined

$$
\eta_{ \pm}=\frac{x^{\prime}-V t^{\prime} \pm b\left(1+t^{\prime} / T\right)}{\sqrt{2 \hbar t^{\prime} / i m} \sqrt{1+t^{\prime} / T}}
$$

and $V=X / T$. The probability density is the absolute square of this, so

$$
P\left(x^{\prime}+X, t^{\prime}+T ; 0,0\right)=\left|K\left(x^{\prime}+X, t^{\prime}+T ; 0,0\right)\right|^{2}=|C|^{2} \frac{m}{\pi^{2} \hbar T}\left(1+\frac{t^{\prime}}{T}\right)^{-1}\left|\int_{\eta_{-} / i}^{\eta_{+} / i} d w e^{-w^{2}}\right|^{2}
$$

To find the normalization constant $C$ we may use expression 4.1.5. Hence

$$
\begin{aligned}
& P\left(x^{\prime}+X, t^{\prime}+T ; 0,0\right)=K\left(x^{\prime}+X, t^{\prime}+T ; 0,0\right) K^{*}\left(x^{\prime}+X, t^{\prime}+T ; 0,0\right)=|C|^{2} \frac{m^{2}}{4 \pi^{2} \hbar^{2} t^{\prime} T} \\
& \times\left|\int_{-b}^{b} \int_{-b}^{b} d y d y^{\prime} \exp \left\{\frac{i m}{2 \hbar t^{\prime}}\left(y^{2}+y^{\prime 2}\right)\left(1+\frac{t^{\prime}}{T}\right)\right\} \exp \left\{-\frac{i m}{\hbar t^{\prime}}\left(y+y^{\prime}\right)\left(x^{\prime}-V t^{\prime}\right)\right\}\right|
\end{aligned}
$$

so that the total probability of finding a particle at the screen is

$$
\begin{aligned}
1 & =\int_{-\infty}^{\infty} d x^{\prime} P\left(x^{\prime}+X, t^{\prime}+T ; 0,0\right) \\
& =|C|^{2} \frac{m^{2}}{2 \pi \hbar^{2} t^{\prime} T}\left|\int_{-b}^{b} \int_{-b}^{b} d y d y^{\prime} \delta\left(\frac{m\left(y+y^{\prime}\right)}{\hbar t^{\prime}}\right) \exp \left\{\frac{i m}{2 \hbar t^{\prime}}\left(y^{2}+y^{\prime 2}\right)\left(1+\frac{t^{\prime}}{T}\right)+\frac{1}{2}\left(y+y^{\prime}\right) V t^{\prime}\right\}\right|
\end{aligned}
$$

where $\delta(z)$ is the Dirac delta distribution. By using the property

$$
\delta(k z)=\frac{1}{|k|} \delta(z)
$$


we find for 4.1.11

$$
1=|C|^{2} \frac{m^{2}}{2 \pi \hbar^{2} t^{\prime} T} \frac{\hbar t^{\prime}}{m}\left|\int_{-b}^{b} d y \exp \left\{\frac{i m}{\hbar t^{\prime}}\left(1+\frac{t^{\prime}}{T}\right) y^{2}\right\}\right|
$$

or that

$$
|C|^{2}=\frac{2 \pi \hbar T}{m}\left|\int_{-b}^{b} d y \exp \left\{\frac{i m}{\hbar t^{\prime}}\left(1+\frac{t^{\prime}}{T}\right) y^{2}\right\}\right|^{-1} .
$$

Thus the normalized propagator for the square slit is

$$
\begin{aligned}
& K\left(x^{\prime}+X, t^{\prime}+T ; 0,0\right)=\sqrt{\frac{2}{\pi i}}\left(1+\frac{t^{\prime}}{T}\right)^{-1 / 2}\left|\int_{-b}^{b} d y \exp \left\{\frac{i m}{\hbar t^{\prime}}\left(1+\frac{t^{\prime}}{T}\right) y^{2}\right\}\right|^{-1 / 2} \\
& \times \exp \left\{\frac{i m}{2 \hbar}\left[\frac{X^{2}}{T}+\frac{x^{\prime 2}}{t^{\prime}}+\frac{1}{t^{\prime}}\left(x^{\prime}-V t^{\prime}\right)\left(1+\frac{t^{\prime}}{T}\right)^{-1}\right]\right\} \int_{\eta_{-} / i}^{\eta_{+} / i} d w e^{-w^{2}}
\end{aligned}
$$

We wish to end the analysis here since when we are considering the Gaussian slit, the results will we the same as for the Gaussian slit and the mathematics will lead us more quickly to the physics.

\subsubsection{Single Slit of Gaussian Type}

In this section, we are going to extend the setup of the square slit by introducing a function that is dependent on the displacement along the slit. In the case of the square slit, we can not find a closed form expression of the propagator in terms of elementary functions, so we are redirected to the method of approximation. In contrast to the mathematical complexities arising from the analysis of the square slit, the Gaussian shaped slit takes us quickly to the physics, as will be shown. A general form of a Gaussian function is

$$
f(y)=\exp \left\{\frac{-y^{2}}{2 b^{2}}\right\}
$$

where $y$ is the integration variable and $2 b$ the width of the slit. The constant $b$ is also known as the standard deviation and it is the half-width of the curve from the center to where the height of the curve has dropped by a factor of $e^{-1}$. If the shape of the slit is such that most of the area lies within the width $2 b$ of the bell-shaped curve we can with small errors extend the integration interval from $(-b, b)$ to $(-\infty, \infty)$ and solve a Gaussian integral for which we know a closed form. So if we multiply the integrand in the square slit integral by a function $f(y)$ we obtain the expression for the Gaussian slit propagator as

$$
\begin{aligned}
K\left(x^{\prime}+X, t^{\prime}+\right. & T ; 0,0)=C \frac{m}{2 \pi i \hbar \sqrt{t^{\prime} T}} \int_{-\infty}^{\infty} d y f(y) \exp \left\{\frac{i m}{2 \hbar}\left[\frac{(X+y)^{2}}{T}+\frac{\left(x^{\prime}-y\right)^{2}}{t^{\prime}}\right]\right\} \\
& =C \frac{m}{2 \pi i \hbar \sqrt{t^{\prime} T}} \int_{-\infty}^{\infty} d y \exp \left\{-\frac{y^{2}}{2 b^{2}}\right\} \exp \left\{\frac{i m}{2 \hbar}\left[\frac{(X+y)^{2}}{T}+\frac{\left(x^{\prime}-y\right)^{2}}{t^{\prime}}\right]\right\}
\end{aligned}
$$

where we have included a constant $C$ since introducing the function $f(y)$ will leave the propagator not normalized. We follow the same ideas as for the single square slit. First we complete the square in the exponent as

$$
-\frac{y^{2}}{2 b^{2}}+\frac{i m}{2 \hbar}\left[\frac{(X+y)^{2}}{T}+\frac{\left(x^{\prime}-y\right)^{2}}{t^{\prime}}\right]=\frac{i m}{2 \hbar}\left[\frac{x^{2}}{T}+\frac{x^{\prime 2}}{t^{\prime}}+A\left(y+\frac{B}{A}\right)^{2}-\frac{B^{2}}{A}\right]
$$




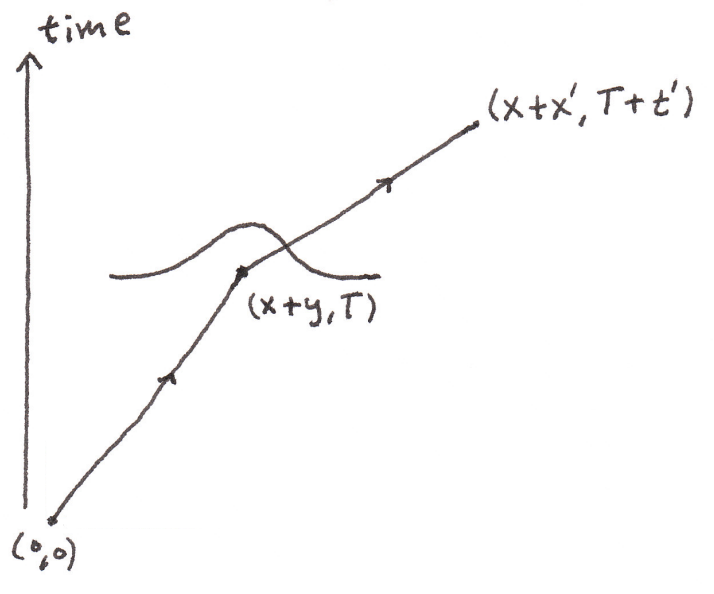

Figure 3: A free propagation through a Gaussian slit. The particle moves to a point $(x+y, T)$ where its motion is restricted by the Gaussian slit and moves further to a point $\left(x+x^{\prime}, T+t^{\prime}\right)$. The shape of the slit enable us to stretch the integration limits to $-\infty$ and $\infty$ with very small errors since the contributions to the amplitude will be negligible.

where we have defined the constants

$$
\begin{aligned}
A & =\frac{i \hbar}{m b^{2}}+\frac{1}{T}+\frac{1}{t^{\prime}}, \\
B & =\frac{X}{T}-\frac{x^{\prime}}{t^{\prime}} .
\end{aligned}
$$

Then if we substitute this into 4.1.16 we get

$$
\begin{aligned}
K\left(x^{\prime}+X, t^{\prime}+T ; 0,0\right) & =C \frac{m}{2 \pi i \hbar \sqrt{t^{\prime} T}} \\
& \times \exp \left\{\frac{i m}{2 \hbar}\left[\frac{X^{2}}{T}+\frac{x^{\prime 2}}{t^{\prime}}-\frac{B^{2}}{A}\right]\right\} \int_{-\infty}^{\infty} d y \exp \left\{\frac{i m A}{2 \hbar}\left(y+\frac{B}{A}\right)^{2}\right\} \\
& =C \sqrt{\frac{2 \pi i \hbar}{m A}} \frac{m}{2 \pi i \hbar \sqrt{t^{\prime} T}} \exp \left\{\frac{i m}{2 \hbar}\left[\frac{X^{2}}{T}+\frac{x^{\prime 2}}{t^{\prime}}-\frac{B^{2}}{A}\right]\right\} \\
& =C \sqrt{\frac{m}{2 \pi i \hbar A t^{\prime} T}} \exp \left\{\frac{i m}{2 \hbar}\left[\frac{X^{2}}{T}+\frac{x^{\prime 2}}{t^{\prime}}-\frac{A^{*} B^{2}}{|A|^{2}}\right]\right\},
\end{aligned}
$$

where we have multiplied and divided the last term in the exponential by the complex conjugate of $A, A^{*}$. Then if we absolute square 4.1 .19 we get

$$
\begin{aligned}
& P\left(X+x^{\prime}, T+t^{\prime} ; 0,0\right)=\left|K\left(X+x^{\prime}, T+t^{\prime} ; 0,0\right)\right|^{2} \\
& =\left|C \sqrt{\frac{m}{2 \pi i \hbar A t^{\prime} T}}\right|^{2}\left|\exp \left\{\frac{i m}{2 \hbar}\left[\frac{X^{2}}{T}+\frac{x^{\prime 2}}{t^{\prime}}\right]\right\}\right|^{2}\left|\exp \left\{\frac{-i m A^{*} B^{2}}{2 \hbar|A|^{2}}\right\}\right|^{2} \\
& =|C|^{2} \frac{m}{2 \pi \hbar|A| t^{\prime} T}\left|\exp \left\{\frac{-i m A^{*} B^{2}}{2 \hbar|A|^{2}}\right\}\right|^{2} .
\end{aligned}
$$

If we expand the argument

$$
\frac{-i m A^{*} B^{2}}{2 \hbar|A|^{2}}=\frac{-i m}{2 \hbar} \frac{\left(-i \hbar / m b^{2}+1 / T+1 / t^{\prime}\right)\left(X / T-x^{\prime} / t^{\prime}\right)^{2}}{\hbar^{2} /\left(m^{2} b^{4}\right)+\left(1 / T+1 / t^{\prime}\right)^{2}} .
$$

we can see that the only term surviving is the first one on the right hand side in (4.1.21) in the exponent hence we get

$$
\frac{-1 / 2 b^{2}\left(X / T-x^{\prime} / t^{\prime}\right)^{2}}{\hbar^{2} /\left(m^{2} b^{4}\right)+\left(1 / T+1 / t^{\prime}\right)^{2}} \text {. }
$$


If we substitute our result in 4.1 .19 the probability becomes

$$
\begin{aligned}
P\left(X+x^{\prime}, T+t^{\prime} ; 0,0\right) & =|C|^{2} \frac{m}{2 \pi \hbar|A| t^{\prime} T} \exp \left\{\frac{-\left(X / T-x^{\prime} / t^{\prime}\right)^{2}}{\hbar^{2}\left(m^{2} b^{2}\right)+\left(1 / T+1 / t^{\prime}\right)^{2}}\right\} \\
& =|C|^{2} \frac{m}{2 \pi \hbar|A| t^{\prime} T} \exp \left\{\frac{-\left(x^{\prime}-V t^{\prime}\right)^{2}}{(\delta x)^{2}}\right\},
\end{aligned}
$$

where we have defined the quantities

$$
\begin{aligned}
X & =V T \\
(\delta x)^{2} & =\frac{\hbar^{2} t^{\prime 2}}{m^{2} b^{2}}+b^{2}\left(\frac{t^{\prime}}{T}+1\right)^{2},
\end{aligned}
$$

and $V$ is the average velocity of the particle. The quantity $(\delta x)^{2}$ tell us the effective width of the distribution as detected some distance away from the slit. The second term is what we would expect to see classically since if we increase the time $t^{\prime}$ the width of the distribution increases. On smaller scales, however, this is not what we would see experimentally but the width is actually greater than expected. This can be explained by the first term and the effective width is obviously a quantum mechanical effect because of the appearance of Planck's constant $\hbar$. If we integrate 4.1.23 over all $x^{\prime}$ we get the probability of the system to be in any state at the screen as

$$
\begin{aligned}
1=\int_{-\infty}^{\infty} d x^{\prime} P\left(x^{\prime}, t^{\prime} ; 0,0\right) & =|C|^{2} \frac{m}{2 \pi \hbar|A| t^{\prime} T} \int_{-\infty}^{\infty} d x^{\prime} \exp \left\{-\frac{\left(x^{\prime}-V t^{\prime}\right)^{2}}{(\delta x)^{2}}\right\} \\
& =|C|^{2} \frac{m}{2 \pi \hbar|A| t^{\prime} T} \sqrt{(\delta x)^{2} \pi}
\end{aligned}
$$

Solving for $|C|^{2}$ gives, apart from a phase,

$$
|C|^{2}=\frac{2 \sqrt{\pi} \hbar T}{m b}
$$

thus 4.1 .23 becomes

$$
\begin{aligned}
P\left(X+x^{\prime}, T+t^{\prime} ; 0,0\right) & =\frac{2 \sqrt{\pi} \hbar T}{m b} \frac{m}{2 \pi \hbar|A| t^{\prime} T} \exp \left\{\frac{-\left(x^{\prime}-V t^{\prime}\right)^{2}}{(\delta x)^{2}}\right\} \\
& =\frac{1}{\sqrt{\pi}(\delta x)} \exp \left\{\frac{-\left(x^{\prime}-V t^{\prime}\right)^{2}}{(\delta x)^{2}}\right\}
\end{aligned}
$$

where we have used the definition of $A$ to write

$$
|A|^{2} b^{2} t^{\prime 2}=(\delta x)^{2} .
$$

If we use a hand-wave analysis of the probability 4.1 .25 by not introducing the constant $C$, the probability of the system to be in any state at the screen is

$$
\int_{-\infty}^{\infty} d x^{\prime} P\left(X+x^{\prime}, T+t^{\prime} ; 0,0\right)=\frac{m}{2 \pi \hbar T} b \sqrt{\pi}
$$

We can see that the integral over the probability over all $x^{\prime}$ is independent of the time $t^{\prime}$ as expected. It is dependent on the mass $m$ and the average time of travel $T$ of the particle. Let us now ignore for the moment that this has dimension of $1 / L$. This result can with no problem be compared to the exactly same result that the probability that the particle hits the Gaussian slit within the interval $\Delta y$ in the range of the slit. If $\Delta y$ is small we find that

$$
\begin{aligned}
P(X+y, T ; 0,0) \Delta y & =|K(X+y, T ; 0,0)|^{2} \Delta y \\
& =\left|\sqrt{\frac{m}{2 \pi i \hbar T}}\right|^{2}\left|\exp \left\{\frac{i m}{2 \hbar T}(X+y)^{2}\right\}\right|^{2} \Delta y \\
& =\frac{m}{2 \pi \hbar T} \Delta y .
\end{aligned}
$$


The probability that the particle enter through the slit must be exactly the same as detecting the particle after passing through it. Then 4.1.29 and 4.1.30 are equal and the effective width of the Gaussian slit is $b \sqrt{\pi}$.

\subsubsection{Double Slit of a Gaussian Type}

The treatment of the single Gaussian slit can be extended to multiple slits allowed to interact with each other. We are going to use the principle of superposition for the double slit of Gaussian type by simply adding them together and obtain the total propagator for the system. One should see that the probability distribution contains an additional term that contains information for the interference between the both slit configurations. Say we simply add another slit to the configuration of the single Gaussian slit above. Call the propagator of one of the slits $K_{1}$ and the other for $K_{2}$ then from the principle of superposition we shall add $K_{1}$ to $K_{2}$ to obtain the final propagator $K$ as

$$
K=K_{1}+K_{2} \text {. }
$$

The complex nature of the propagator will output the probability density

$$
|K|^{2}=\left|K_{1}+K_{2}\right|^{2}=\left|K_{1}\right|^{2}+\left|K_{2}\right|^{2}+2 \operatorname{Re}\left(K_{1}^{*} K_{2}\right)
$$

where the first two terms, corresponding to each slit with no interaction, gives a similar result that to 4.1.23 but we are interested in the last term. It contains information about the interaction of the two systems and is mainly what we are going to treat here.

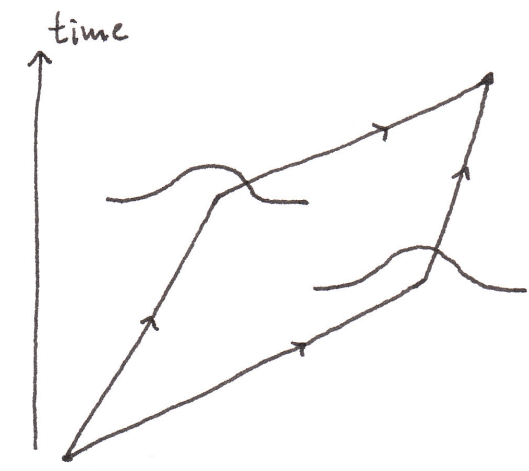

Figure 4: The configuration of the two Gaussian slits with amplitudes shown for each slit. We have added a similar slit to allow interference between two slits so we obtain an interaction term in the probability density.

The propagator $K$ can be written as

$$
K=K_{1}+K_{2}=K_{1}\left(X_{1}+x^{\prime}, T_{1}+t^{\prime} ; 0,0\right)+K_{2}\left(X_{2}+x^{\prime}, T_{2}+t^{\prime} ; 0,0\right)
$$

where the coordinates are chosen just in analogy with the single Gaussian slit. If we now use the result of 4.1.19 we get the expression

$$
\begin{aligned}
K & =\sqrt{\frac{m}{2 \pi i \hbar A_{1} T_{1} t^{\prime}}} \exp \left\{\frac{i m}{2 \hbar}\left[\frac{X_{1}^{2}}{T_{1}}+\frac{x^{\prime 2}}{t^{\prime}}-\frac{A_{1}^{*} B_{1}^{2}}{\left|A_{1}\right|^{2}}\right]\right\} \\
& +\sqrt{\frac{m}{2 \pi i \hbar A_{2} T_{2} t^{\prime}}} \exp \left\{\frac{i m}{2 \hbar}\left[\frac{X_{2}^{2}}{T_{2}}+\frac{x^{\prime 2}}{t^{\prime}}-\frac{A_{2}^{*} B_{2}^{2}}{\left|A_{2}\right|^{2}}\right]\right\}
\end{aligned}
$$

where we have defined

$$
\begin{aligned}
& A_{j}=\frac{i \hbar}{m b_{j}{ }^{2}}+\frac{1}{T_{j}}+\frac{1}{t^{\prime}} \\
& B_{j}=\frac{X_{j}}{T_{j}}-\frac{x^{\prime}}{t^{\prime}}, \quad j=1,2 .
\end{aligned}
$$


Next we find the probability density of the total system by absolute squaring (4.1.34). Since we already know the two densities for each individual system from the single slit case we are focusing on the third term on the right hand side of 4.1 .32 . The interference part can be written as

$$
\operatorname{Re}\left(K_{1}^{*} K_{2}\right) \propto \cos \left\{\frac{m}{2 \hbar}\left[\frac{X_{2}^{2}}{T_{2}}-\frac{X_{1}^{2}}{T_{1}}+\frac{A_{1} B_{1}^{2}}{\left|A_{1}\right|^{2}}-\frac{A_{2}^{*} B_{2}^{2}}{\left|A_{2}\right|^{2}}\right]\right\}
$$

where the indicies denotes each slit. By using 4.1.28) and the definition of $B_{j}$ we can rewrite the second term in the argument of the cosine as

$$
\frac{A_{1} B_{1}^{2}}{\left|A_{1}\right|^{2}}-\frac{A_{2}^{*} B^{2}}{\left|A_{2}\right|^{2}}=\frac{A_{1} b_{1}^{2}\left(x^{\prime}-V_{1} t^{\prime}\right)^{2}}{\left(\delta x_{1}\right)^{2}}-\frac{A_{2}^{*} b_{2}^{2}\left(x^{\prime}-V_{2} t^{\prime}\right)^{2}}{\left(\delta x_{2}\right)^{2}}
$$

where we have used the same analogy as for the single Gaussian slit where $\delta x_{j}$ is the effective distribution width for the slit $j$ and $V_{j}=X_{j} / T_{j}$ is the average velocity of the particles before the respective slit. If we pass the limit of either slit to become similar to the other, such that distances, velocities etc. of either slit becomes equal to each other, this term reduces to

$$
\begin{aligned}
& {\left[\frac{A_{1} b_{1}^{2}\left(x^{\prime}-V_{1} t^{\prime}\right)^{2}}{\left(\delta x_{1}\right)^{2}}-\frac{A_{2}^{*} b_{2}^{2}\left(x^{\prime}-V_{2} t^{\prime}\right)^{2}}{\left(\delta x_{2}\right)^{2}}\right]} \\
& \rightarrow \frac{b^{2}\left(x^{\prime}-V t^{\prime}\right)^{2}}{(\delta x)^{2}}\left[A-A^{*}\right]=\frac{2 i \hbar\left(x^{\prime}-V t^{\prime}\right)^{2}}{m(\delta x)^{2}} .
\end{aligned}
$$

The first term in the cosine vanishes when we pass the limit and the interaction part becomes

$$
\cos \left\{\frac{i\left(x^{\prime}-V t^{\prime}\right)^{2}}{(\delta x)^{2}}\right\}=\cosh \left\{\frac{\left(x^{\prime}-V t^{\prime}\right)^{2}}{(\delta x)^{2}}\right\} .
$$

Thus the resulting interfering part are a hyperbolic cosine that is the sum of the exponential 4.1.23 and another equal exponential but with opposite sign. This means that there are no possibility for completely destructive interference, because the hyperbolic cosine is always larger than zero unless its argument is imaginary and this would result in some of the observables to be imaginary which makes no sense.

\subsection{The Energies for the Quantum Harmonic Oscillator}

In this section, we are going to show how to obtain the energies for the harmonic oscillator ${ }^{8}$ using the path integral. The main idea is to integrate the propagator (3.8.3) and extract the time-dependent terms including the energies $E_{n}$. Then we perform the same integration but replacing (3.8.3) with the explicit from of the harmonic oscillator propagator, express the result as a series expansion and compare it with 3.8 .3 . From the uniqueness of the series expansion we can compare the terms in the series on each side and identify all energies. The propagator for the harmonic oscillator i:9

$$
\begin{aligned}
& K\left(x, t ; x^{\prime}, t^{\prime}\right)= \\
& \quad \sqrt{\frac{m \omega}{2 \pi i \hbar \sin \left(\omega\left(t-t^{\prime}\right)\right)}} \exp \left\{\frac{i m \omega}{2 \hbar \sin \left(\omega\left(t-t^{\prime}\right)\right)}\left[\left(x^{\prime 2}+x^{2}\right) \cos \left(\omega\left(t-t^{\prime}\right)\right)-2 x^{\prime} x\right]\right\}
\end{aligned}
$$

where $t>t^{\prime}$ and it is understood that this expression is not valid when $\omega t=2 \pi n$ where $n$ is an integer. From (3.8.3) we can express the propagator in terms of the solutions to the Schrödinger equation, restated here for convenience, as

$$
K\left(x, t ; x^{\prime}, t^{\prime}\right)=\sum_{n=0}^{\infty} X_{n}^{*}\left(x^{\prime}\right) X_{n}(x) \exp \left(-i E_{n}\left(t-t^{\prime}\right) / \hbar\right)
$$

\footnotetext{
${ }^{8}$ See the appendix, section 3

${ }^{9}$ See the Appendix, Section 2
} 
where $t>t^{\prime}$. Now we wish to extract the time dependence from the propagator and by letting $x^{\prime}=x$ and $t^{\prime}=0$ in the propagator and integrating over all $x$ we get

$$
\begin{aligned}
\int_{-\infty}^{\infty} d x K(x, t ; x, 0) & =\int_{-\infty}^{\infty} d x \sum_{n=0}^{\infty} X_{n}^{*}(x) X_{n}(x) \exp \left(-i E_{n} t / \hbar\right) \\
& =\sum_{n=0}^{\infty} \exp \left(-i E_{n} t / \hbar\right) \int_{-\infty}^{\infty} d x X_{n}^{*}(x) X_{n}(x)=\sum_{n=0}^{\infty} \exp \left(-i E_{n} t / \hbar\right)
\end{aligned}
$$

where we have used the result from A.1.24 for the integration and now we perform the same integration with the explicit form of the propagator for the harmonic oscillator:

$$
\begin{aligned}
\int_{-\infty}^{\infty} d x K(x, t ; x, 0) & =\int_{-\infty}^{\infty} d x \sqrt{\frac{m}{2 \pi i \hbar \sin (\omega t)}} \exp \left\{\frac{i m \omega}{\hbar \sin (\omega t)}\left[(\cos (\omega t)-1) x^{2}\right]\right\} \\
& =\sqrt{\frac{m}{2 \pi i \hbar \sin (\omega t)}} \int_{-\infty}^{\infty} d x \exp \left\{-\frac{2 i m \omega}{\hbar \sin (\omega t)} \sin ^{2}\left(\frac{\omega t}{2}\right) x^{2}\right\}
\end{aligned}
$$

The integral is a Gaussian and can be easily solved and then we get

$$
\begin{aligned}
\int_{-\infty}^{\infty} d x K(x, t ; x, 0) & =\sqrt{\frac{m \omega}{2 \pi i \hbar \sin (\omega t)}} \sqrt{\frac{\pi \hbar \sin (\omega t)}{2 i m \omega}}\left[\sin ^{2}\left(\frac{\omega t}{2}\right)\right]^{-1 / 2} \\
& =\frac{1}{2 i}\left[\sin ^{2}\left(\frac{\omega t}{2}\right)\right]^{-1 / 2}=\frac{1}{2 i}\left[\sin \left(\frac{\omega t}{2}\right)\right]^{-1} .
\end{aligned}
$$

By using the definition of the sine function

$$
\sin (k t)=\frac{\exp (i k t)-\exp (-i k t)}{2 i}
$$

from complex analysis we can re-write 4.2 .5 as

$$
\frac{1}{2 i}\left[\sin \left(\frac{\omega t}{2}\right)\right]^{-1}=\frac{1}{\exp (i \omega t / 2)-\exp (-i \omega t / 2)}=\frac{\exp (-i \omega t / 2)}{1-\exp (i \omega t)} .
$$

By comparing this with 4.2 .7 we clearly see that the series is divergent for $|\exp (i \omega t)|=1$ and we get a pole. We can work around this by shifting the exponential by a small complex number $\eta$ such that the condition $|\exp (i \omega t)|<1$ is valid, re-write the denominator factor as a converging sum and then let $\eta \rightarrow 0$ to obtain

$$
\frac{\exp (-i \omega t / 2)}{1-\exp (i \omega t)}=\exp (-i \omega t / 2) \sum_{n=0}^{\infty} \exp (-i n \omega t)=\sum_{n=0}^{\infty} \exp [-i \omega(n+1 / 2) t]
$$

By comparing this series to 4.2 .3 we get the energies for the quantum harmonic oscillator as

$$
E_{n}=\left(n+\frac{1}{2}\right) \hbar \omega
$$

where $n$ is an integer.

\subsection{The Aharonov-Bohm Effect}

The Aharonov-Bohm effect is a great example of how the magnetic vector potential in classical electrodynamics plays an independent role in quantum mechanics, meaning that in classical electrodynamics we only use the vector potential as a tool, whereas the rotation of it, the $B$-field, has the physical meaning. In quantum mechanics the vector potential itself have a physical meaning 


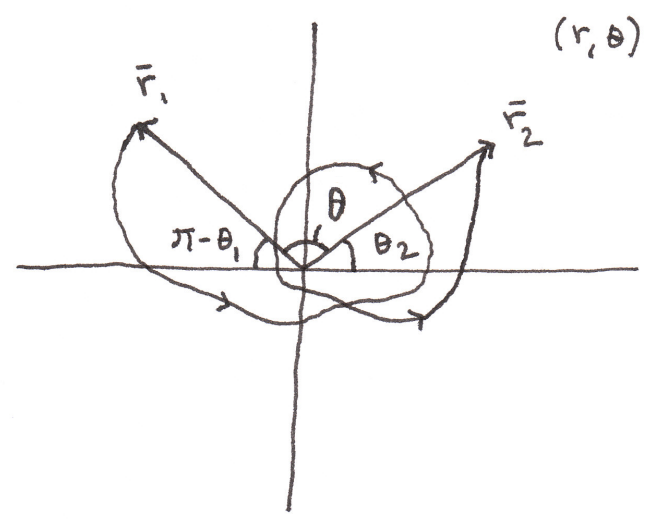

Figure 5: Illustration of the experimental setup. The source of particles origins from the point $\boldsymbol{r}_{1}$ and finishes the journey at the point $\boldsymbol{r}_{2}$. The view is such that we are looking down, or up, along the infinitesimally thin and long Dirac string. The angles for $\boldsymbol{r}_{1}$ and $\boldsymbol{r}_{2}$ are also visible, as well as the angle $\theta$ between them.

as we will later find out. The experiment that exposes the physical independence of the vector potential can be illustrated as follows: Inside a cylinder there exists a magnetic field $\boldsymbol{B}$. The cylinder is isolated such that a particle, bypassing the cylinder on the outside, can not be effected by the magnetic field inside. Even though the magnetic field is zero on the outside of the cylinder, the magnetic vector potential $\boldsymbol{A}$ is non-zero there. This is in agreement with classical theory.

Now we are shrinking the radius of the cylinder such that we obtain an infinitely thin and infinitely long string at the origo in the polar plane that we will call a Dirac string, "containing" the magnetic field. The particles leaves a source at a point $\boldsymbol{r}_{1}$ and ends up at a point $\boldsymbol{r}_{2}$, and are not allowed to cross the origin. Mathematically, we introduce an expression for each possible path a particle might take, that when summed up gives the probability amplitude given the angular difference $\phi=\theta_{2}-\theta_{1}+2 \pi n$ between $\boldsymbol{r}_{1}$ and $\boldsymbol{r}_{2}$. The propagator is given by

$$
K\left(\boldsymbol{r}_{2}, t_{2} ; \boldsymbol{r}_{1}, t_{1}\right)=\int_{-\infty}^{\infty} d \phi K_{\phi}
$$

where 10

$$
\begin{aligned}
K_{\phi}\left(\boldsymbol{r}_{2}, t_{2} ; \boldsymbol{r}_{1}, t_{1}\right) & =\frac{m}{2 \pi i \hbar\left(t_{2}-t_{1}\right)} \sum_{n=-\infty}^{\infty} \delta\left(\theta_{2}-\theta_{1}+\phi+2 \pi n\right) \\
& \times \exp \left\{\frac{i m}{2 \hbar} \frac{\left(r_{2}^{2}+r_{1}^{2}\right)}{t_{2}-t_{1}}\right\} \int_{-\infty}^{\infty} d \lambda \exp (-i \phi \lambda) I_{\lambda}\left(\frac{-i m r_{1} r_{2}}{\hbar\left(t_{2}-t_{1}\right)}\right),
\end{aligned}
$$

$I_{\lambda}$ is and asymptotic Bessel function and it is understood that $t_{2}>t_{1}$. The delta function ensures that the difference in the polar angles between $\boldsymbol{r}_{1}$ and $\boldsymbol{r}_{1}$ is an integer multiple of $2 \pi$. The Lagrangian used to derive the propagator was

$$
L=\frac{1}{2} m \dot{\boldsymbol{r}}^{2}-\hbar \lambda \dot{\theta}
$$

where the second term on the right hand side comes from the derivation in A.4.10 $\lambda$ is just an integration variable coming from the integral representation of the Dirac delta. If we now introduce a term to the free particle Lagrangian containing a vector potential

$$
L_{\boldsymbol{A}}=q \boldsymbol{A} \cdot \dot{\boldsymbol{r}}
$$

where $q$ is the charge, $\boldsymbol{A}$ the vector potential and $\dot{\boldsymbol{r}}$ the velocity of the charge, the Lagrangian term 4.3.3 changes the Lagrangian $L$ as

$$
L^{\prime}=L+L_{\boldsymbol{A}}=L_{0}+q \boldsymbol{A} \cdot \dot{\boldsymbol{r}}-\hbar \lambda \dot{\theta}
$$

\footnotetext{
${ }^{10}$ We are leaving the derivation, contained in the appendix, section 3 , to the reader since it is rather lengthy.
} 
where we have defined $L_{0}$ to be the Lagrangian of a free charge. Next, we will suggest a form of the vector potential such that it is non-zero outside the origo, but the curl operation on it yields no magnetic field when $r \neq 0$, but non-zero when $r=0$. A magnetic vector potential satisfying these properties could be

$$
\boldsymbol{A}=\frac{\chi}{r} \hat{\boldsymbol{z}} \times \hat{\boldsymbol{r}}
$$

where $\chi$ is a constant. We see that it has an inverse radial dependence and the magnitude gets very large for small $r$ and it is clearly undefined at the origin. The curl operation of this vector potential yields the magnetic field

$$
\boldsymbol{B}=\nabla \times \boldsymbol{A}=\chi \nabla \times\left(r^{-1} \hat{\boldsymbol{z}} \times \hat{\boldsymbol{r}}\right) .
$$

To resolve the right hand side we can use the vector calculus identity

$$
\nabla \times(f \boldsymbol{g})=\nabla f \times \boldsymbol{g}+f \nabla \times \boldsymbol{g}
$$

where $f$ is a scalar and $\boldsymbol{g}$ a vector. If we replace $f$ by $r^{-1}$ and $g$ by $\hat{\boldsymbol{x}} \times \hat{\boldsymbol{r}}$ we find that

$$
\begin{aligned}
\nabla \times\left(r^{-1} \hat{\boldsymbol{z}} \times \hat{\boldsymbol{r}}\right) & =\nabla\left(r^{-1}\right) \times(\hat{\boldsymbol{z}} \times \hat{\boldsymbol{r}})+r^{-1}(\nabla \times(\hat{\boldsymbol{z}} \times \hat{\boldsymbol{r}})) \\
& =-r^{-2} \hat{\boldsymbol{r}} \times(\hat{\boldsymbol{z}} \times \hat{\boldsymbol{r}})+r^{-1} \nabla \times(\hat{\boldsymbol{z}} \times \hat{\boldsymbol{r}}) .
\end{aligned}
$$

The first term on the right hand side is $-r^{-2} \hat{\boldsymbol{z}}$ since $\hat{\boldsymbol{z}} \times \hat{\boldsymbol{r}}=\hat{\boldsymbol{\theta}}$ because we have cylindrical symmetry and we have $\hat{\boldsymbol{r}} \times \hat{\boldsymbol{\theta}}$ which is just $\hat{\boldsymbol{z}}$. The second term on the right hand side is

$$
r^{-1} \nabla \times(\hat{\boldsymbol{z}} \times \hat{\boldsymbol{r}})=\hat{\boldsymbol{z}} r^{-2} \frac{\partial r}{\partial r}=\hat{\boldsymbol{z}} r^{-2}
$$

where we have used the definition of the curl in cylindrical coordinates. By substituting our results for the magnetic field we obtain

$$
\boldsymbol{B}=\nabla \times \boldsymbol{A}=-r^{-2} \hat{\boldsymbol{z}}+r^{-2} \hat{\boldsymbol{z}}=0
$$

which is just the condition for the magnetic field outside the origo and this is of course valid only for $r \neq 0$. The magnetic flux when including the origin, can be written as a line integral evaluated one revolution in the polar plane perpendicular to the Dirac string as

$$
\Phi \equiv \int \boldsymbol{B} \cdot d \boldsymbol{S}=\int \nabla \times \boldsymbol{A} \cdot d \boldsymbol{S}=\oint \boldsymbol{A} \cdot d \boldsymbol{l}=\oint \frac{\chi}{r} \hat{\boldsymbol{z}} \times \hat{\boldsymbol{r}} \cdot r d \theta \hat{\boldsymbol{\theta}}=\int d \theta \chi=2 \pi \chi
$$

where we can see that the constant $\chi$ is proportional to the magnetic flux. Now let us go back to the vector potential term in the Lagrangian $L_{\boldsymbol{A}}$ : In the $(r, \theta)$-plane we have the vectors $\boldsymbol{r}_{1}$ and $\boldsymbol{r}_{2}$ where $\boldsymbol{r}_{i}$ is defined as

$$
\boldsymbol{r}_{i}=r_{i} \cos \left(\theta_{i}\right) \hat{\boldsymbol{x}}+r_{i} \sin \left(\theta_{i}\right) \hat{\boldsymbol{y}} .
$$

The Lagrangian term for the magnetic vector potential contains the time derivative of this vector which implicitly is

$$
\frac{d \boldsymbol{r}}{d t}=\dot{\boldsymbol{r}}=\frac{d r}{d t} \hat{\boldsymbol{r}}+r \frac{d \theta}{d t} \hat{\boldsymbol{\theta}} .
$$

By substituting the definition of $\dot{\boldsymbol{r}}$ in $L_{\boldsymbol{A}}$ we get

$$
\begin{aligned}
L_{\boldsymbol{A}}=q \boldsymbol{A} \cdot \dot{\boldsymbol{r}} & =\frac{q \chi}{r^{2}} \hat{\boldsymbol{z}} \times r \hat{\boldsymbol{r}} \cdot\left(\frac{d r}{d t} \hat{\boldsymbol{r}}+r \frac{d \theta}{d t} \hat{\boldsymbol{\theta}}\right) \\
& =\frac{q \chi}{r} \hat{\boldsymbol{\theta}} \cdot\left(\frac{d r}{d t} \hat{\boldsymbol{r}}+r \frac{d \theta}{d t} \hat{\boldsymbol{\theta}}\right) \\
& =q \chi \dot{\theta} .
\end{aligned}
$$


It turns out that this magnetic vector potential Lagrangian only depends on the angular speed $\dot{\theta}$. So by substituting this in 4.3.5 the Lagrangian $L^{\prime}$ becomes

$$
L=L_{0}+q \chi \dot{\theta}-\hbar \lambda \dot{\theta}
$$

which can be written as

$$
L=L_{0}-\hbar(\lambda-q \chi / \hbar) \dot{\theta} .
$$

From this result we can see that the additional effect of a magnetic potential acting on the charges produces a shift $\lambda \rightarrow \lambda-q \chi / \hbar$ in the propagator. So if we look back to the propagator 4.3.2 and the times as $t_{1}=0$ and $t_{2}=\tau$ and shift $\lambda \rightarrow \lambda-q \chi / \hbar$ we get for the integral instead

$$
\int_{-\infty}^{\infty} d \lambda \exp \{-i \phi(\lambda-q \chi / \hbar)\} I_{\lambda-q \chi / \hbar}\left(\frac{-i m r_{1} r_{2}}{\hbar \tau}\right)
$$

and the propagator $K_{\phi}$ will instead have the form

$$
\begin{aligned}
K_{\phi} & =\frac{m}{2 \pi i \hbar \tau} \exp \left\{\frac{i m}{2 \hbar \tau}\left(r_{2}^{2}+r_{1}^{2}\right)\right\} \\
& \times \sum_{n=-\infty}^{\infty} \delta\left(\theta_{2}-\theta_{1}+\phi+2 \pi n\right) \int_{-\infty}^{\infty} d \lambda \exp \{-i \phi(\lambda-q \chi / \hbar)\} I_{\lambda-q \chi / \hbar}\left(-\frac{i m r_{2} r_{1}}{\hbar \tau}\right) .
\end{aligned}
$$

If we now integrate this over all possible values of $\phi$ the Dirac delta function selects out the contributing terms and we obtain

$$
\begin{aligned}
K\left(\boldsymbol{r}_{2}, \tau ; \boldsymbol{r}_{1}, 0\right) & =\frac{m}{2 \pi i \hbar \tau} \exp \left\{\frac{i m}{2 \hbar \tau}\left(r_{2}^{2}+r_{1}^{2}\right)\right\} \\
& \times \sum_{n=-\infty}^{\infty} \int_{-\infty}^{\infty} d \lambda \exp \left\{-i\left(\theta_{2}-\theta_{1}+2 \pi n\right)(\lambda-q \chi / \hbar)\right\} I_{\lambda-q \chi / \hbar}\left(-\frac{i m r_{1} r_{2}}{\hbar \tau}\right),
\end{aligned}
$$

which is the propagator in terms of the winding numbers $n$ and the constant $\chi$. Next, we would like to resolve the integral appearing in the propagator, but the problem in doing so involves the integration over a Bessel function, that is dependent on the integration variable. As most of the contribution to the propagator lies in the classical limit, that is, the fluctuating paths near the classical path makes the significant contributions, we can analyze the propagator in the limit of $\hbar \rightarrow 0$. The argument of the Bessel function inside the integral above, is assumed to be very small in this classical limit. Therefore we can terminate the Bessel function after some terms, that is, neglect terms higher than first order in the argument. Then the asymptotic Bessel function, given from 4.3.20, can be written as

$$
\begin{aligned}
& I_{\lambda-q \chi / \hbar}\left(-\frac{i m r_{1} r_{2}}{\hbar \tau}\right) \approx \\
& \left(\frac{i \hbar \tau}{2 \pi m r_{1} r_{2}}\right)^{1 / 2} \exp \left\{\frac{-i m r_{1} r_{2}}{\hbar \tau}-\frac{1}{2}\left[(\lambda-q \chi / \hbar)^{2}-\frac{1}{4}\right] \frac{i \hbar \tau}{m r_{1} r_{2}}\right\} .
\end{aligned}
$$

By substitution of this approximation of the asymptotic Bessel function in the integral in 4.3.20 we get

$$
\begin{aligned}
\Upsilon \equiv\left(\frac{i \hbar \tau}{2 \pi m r_{1} r_{2}}\right)^{1 / 2} \int_{-\infty}^{\infty} & d \lambda \exp \{-i(\Delta \theta+2 \pi n)(\lambda-q \chi / \hbar)\} \\
& \times \exp \left\{\frac{-i m r_{1} r_{2}}{\hbar \tau}-\frac{1}{2}\left[(\lambda-q \chi / \hbar)^{2}-\frac{1}{4}\right] \frac{i \hbar \tau}{m r_{1} r_{2}}\right\} .
\end{aligned}
$$


This can be re-written as

$$
\begin{aligned}
\left(\frac{i \hbar \tau}{2 \pi m r_{1} r_{2}}\right)^{1 / 2} & \exp \left\{\frac{i q \chi(\Delta \theta+2 \pi n)}{\hbar}+\frac{i q^{2} \chi^{2} \tau}{2 \hbar m r_{1} r_{2}}-\frac{i m r_{1} r_{2}}{\hbar \tau}+\frac{i \hbar \tau}{8 m r_{1} r_{2}}\right\} \\
& \times \exp \left\{\frac{i \hbar \tau}{2 m r_{1} r_{2}}\left(q \chi-\frac{m r_{1} r_{2}(\Delta \theta+2 \pi n)}{\hbar \tau}\right)^{2}\right\} \int_{-\infty}^{\infty} d \lambda \exp \left\{-\frac{i \hbar \tau}{2 m r_{1} r_{2}}(\lambda-c)^{2}\right\}
\end{aligned}
$$

where $c=q \chi-(\Delta \theta+2 \pi n) / \hbar \tau$. The integral is just the inverse of the first factor in 4.3 .23$)$ so they cancel out each other and if we neglect all terms linear in $\hbar$ as $\hbar \rightarrow 0$, as they will have a very small contribution, we approximately get

$$
\Upsilon \approx \exp \left\{i\left(\frac{q \chi}{\hbar}-1\right)(\Delta \theta+2 \pi n)+\frac{i m r_{1} r_{2}}{2 \hbar \tau}(\Delta \theta+2 \pi n)^{2}+\frac{i q^{2} \chi^{2} \tau}{2 \hbar m r_{1} r_{2}}-\frac{i m r_{1} r_{2}}{\hbar \tau}\right\}
$$

If we substitute the result in 4.3.20 we obtain for the propagator $K$

$$
\begin{aligned}
& K\left(\boldsymbol{r}_{2}, \tau ; \boldsymbol{r}_{1}, 0\right)=\frac{m}{2 \pi i \hbar \tau} \exp \left\{\frac{i m}{2 \hbar} \frac{\left(r_{2}-r_{1}\right)^{2}}{\tau}\right\} \times \exp \left\{\frac{i q^{2} \chi^{2} \tau}{2 \hbar m r_{1} r_{2}}\right\} \\
& \times \sum_{n=-\infty}^{\infty} \exp \left\{\frac{i m r_{1} r_{2}}{2 \hbar \tau}(\Delta \theta+2 \pi n)^{2}+i\left(\frac{q \chi}{\hbar}-1\right)(\Delta \theta+2 \pi n)\right\} .
\end{aligned}
$$

For the particle to pass clockwise in a plane seen from above the cylinder the winding number $n$ must be 0 and if the particle pass counter-clockwise the winding number is -1 . Let us then, for simplicity, neglect the particles that passes around the cylinder, having winding numbers $n$ other than $n=0,-1$. Then the propagator reduces to

$$
\begin{aligned}
& K_{0,-1}\left(\boldsymbol{r}_{2}, \tau ; \boldsymbol{r}_{1}, 0\right)=K_{0}+K_{-1}=\frac{m}{2 \pi i \hbar \tau} \exp \left\{\frac{i m}{2 \hbar \tau}\left(r_{2}-r_{1}\right)^{2}\right\} \times \exp \left\{\frac{i q^{2} \chi^{2} \tau}{2 \hbar m r_{1} r_{2}}\right\} \\
& \times\left[\exp \left\{\frac{i m r_{1} r_{2}}{2 \hbar \tau}(\Delta \theta)^{2}+i\left(\frac{q \chi}{\hbar}-1\right) \Delta \theta\right\}+\exp \left\{\frac{i m r_{1} r_{2}}{2 \hbar \tau}(\Delta \theta-2 \pi)^{2}+i\left(\frac{q \chi}{\hbar}-1\right)(\Delta \theta-2 \pi)\right\}\right]
\end{aligned}
$$

where the subscript denotes which terms in the expansion we are considering. The first term corresponds to the winding number $n=0$ and the second term corresponds to the winding number $n=-1$. Classically, there would be no effect since the magnetic field is perfectly isolated and the vector potential is non-physical. But as one can easily see, the wave function will pick up a phase, containing the constant $\chi$ which is precisely the flux through a loop around the cylinder. In other words, in quantum theory, there must be some non-classical mechanism that mediates information that would otherwise not be obtained. We can therefore conclude that the vector potential must have a physical meaning in a quantum theory. We wish to see what happens if we travel along the path corresponding to the winding number $n=-1$ with an initial state $\psi\left(\boldsymbol{r}_{1}, 0\right)$ to a state $\psi\left(\boldsymbol{r}_{2}, \tau\right)$ in a time $\tau$ and then back to the state by the path with winding number $n=0$ in an equal time. The propagator for the entire process is

$$
K_{0}\left(\boldsymbol{r}_{1}, 2 \tau ; \boldsymbol{r}_{2}, \tau\right) K_{-1}\left(\boldsymbol{r}_{2}, \tau ; \boldsymbol{r}_{1}, 0\right)=K_{0}^{*}\left(\boldsymbol{r}_{1}, 0 ; \boldsymbol{r}_{2}, \tau\right) K_{-1}\left(\boldsymbol{r}_{2}, \tau ; \boldsymbol{r}_{1}, 0\right)
$$

where the complex conjugate of $K_{0}$ undoes whatever $K_{-1}$ did, and we must return to the initial state apart from a phase factor. By the definition of how we compose propagators, and since we must return to the initial state, the product on the right hand side of 4.3.27) can be though of as a unit propagator, hence

$$
1=K_{0}^{*}\left(\boldsymbol{r}_{1}, 0 ; \boldsymbol{r}_{2}, \tau\right) K_{-1}\left(\boldsymbol{r}_{2}, \tau ; \boldsymbol{r}_{1}, 0\right)=\exp \left\{\frac{-i 2 \pi q \chi}{\hbar}+\frac{i m r_{1} r_{2}}{2 \hbar \tau}\left[(\Delta \theta-2 \pi)^{2}-(\Delta \theta)^{2}\right]\right\},
$$

apart from a normalization constant. If $2 \hbar \tau>>m r_{1} r_{2}$ or that $\Delta \theta=\pi$, we obtain

$$
q \chi=\hbar k, \quad k=0, \pm 1, \pm 2, \cdots .
$$

Since $\chi$ is constant, the charge $q$ must be quantized in integers of $k$, and this means also that the magnetic flux, which is proportional to $\chi$, must be quantized. 


\section{Conclusion}

The path integral formulation has been shown to be a promising and very useful tool on treating quantum mechanical problems. It is no different from the formulations of, say, Heisenberg, Schrödinger or Dirac since the theories predict the same laws of nature. However all formulations have their place in the quantum theory since it can be advantageous to switch between them. The process of using path integrals can be most difficult and often involves much time to solve them but offers much information about the system even when not being directly solved. The path integral have been proved to be a vital instrument in the development of quantum field theory where it exposes relations between important observables such as in scattering experiments. One also notice that when viewing the equations, a direct relation between classical mechanics and quantum mechanics can be seen, and by specifying a classical theory in terms of Hamiltonian or Lagrangian mechanics, it is armed for any direct quantization.

\section{Acknowledgments}

Many thanks goes to the supervisor for helping out with ideas, stressing the scientific writing language and clarify issues with formulations so they are viewed as clear as possible. 


\section{A Derivation of Propagators}

The theory in this paper does not force the reader to know how the explicit form of the propagators arose but how to use them to obtain physics from them. However in this formulation of quantum mechanics one will constantly be faced upon the path integrals and most of the time have to calculate them explicitly. So for the curious one we here give the derivations of two basic propagators and the more difficult propagator relating winding numbers of the different paths of a system. The basic idea is to use the principle of least action to simplify the evaluation of the action since most of the paths resides very close to the classical path of a system. The propagator for quadratic Lagrangians is therefore the exponential of the classical action.

\section{A.1 The Wave-function in Terms of the Spatial Solutions of the Schrödinger Equation}

There is another way of expressing the propagator where it is written in terms of solutions to the Schrödinger equation. We begin by finding a specific form of the wave-function, the nonrelativistic solutions where the positions and times are independent variables. Let us therefore write the wave-function as a product of a function dependent only on position, where the position does not explicitly or implicitly depend on time, and a function of time only:

$$
\psi(x, t)=X(x) T(t) .
$$

If we substitute this solution in the Schrödinger equation 3.6 .15 we get

$$
i \hbar X(x) \frac{d T(t)}{d t}=-\frac{\hbar^{2}}{2 m} T(t) \frac{d^{2} X(x)}{d x^{2}}+V(x, t) X(x) T(t)
$$

where we have changed the partial derivatives to normal derivatives. The next step is to move the terms dependent only on the position $x$ on one side and the terms dependent on the time $t$ to the other side then we obtain instead

$$
i \hbar \frac{1}{T(t)} \frac{d T(t)}{d t}=-\frac{1}{2 m} \frac{1}{X(x)} \frac{d^{2} X(x)}{d x^{2}}+V(x) .
$$

where we have restricted us only to stationary potentials $V(x)$ dependent only on the position. We see that the left hand side is dependent only on time and the right hand side is dependent only on position. In fact both sides must be equal to a constant since if we make any change to one side the other side remains unaffected by it. Let us set this constant to $E$ and then we get two differential equations

$$
\begin{aligned}
& i \hbar \frac{1}{T(t)} \frac{d T(t)}{d t}=E \\
& \hat{H} X(x)=E X(x) .
\end{aligned}
$$

where $\hat{H}$ is the Hamiltonian operator

$$
\hat{H}=-\frac{\hbar^{2}}{2 m} \frac{d^{2}}{d x^{2}}+V(x) .
$$

The solution to the differential equation in time is

$$
T(t)=\exp (-i E t / \hbar)
$$

Then a solution to the Schrödinger equation can be written as

$$
\psi(x, t)=X(x) T(t)=X(x) \exp (-i E t / \hbar) .
$$


One can also see that the Schrödinger equation is a linear and homogeneous differential equation so if we can find different solutions to the equation then any linear combination of these is itself a solution to the Schrödinger equation. If we write the solutions to A.1.4 as

$$
\begin{aligned}
T_{n}(t) & =\exp \left(-i E_{n} t / \hbar\right), \\
\hat{H} X_{n}(x) & =E_{n} X_{n}(x)
\end{aligned}
$$

where the $n$ 's are real numbers larger than zero and $X_{n}$ are assumed to form a complete set. The general solution to the Schrödinger equation is therefore

$$
\psi(x, t)=\sum_{n} c_{n} X_{n}(x) \exp \left(-i E_{n} t / \hbar\right)
$$

where the $c_{n}$ 's are coefficients left to be determined by a method known as the Fourier's trick by the following: The wave-function A.1.10 with $t$ replaced by $t^{\prime}$ is

$$
\psi\left(x, t^{\prime}\right)=\sum_{n} c_{n} X_{n}(x) \exp \left(-i E_{n} t^{\prime} / \hbar\right)=\sum_{n} a_{n} X_{n}(x)
$$

where we have defined

$$
a_{n}=c_{n} \exp \left(-i E_{n} t^{\prime} / \hbar\right)
$$

Multiplying both sides of A.1.11 by the complex conjugate of $X_{m}(x)$ and integrate over all $x$ gives

$$
\begin{aligned}
\int_{-\infty}^{\infty} d x X_{m}^{*}(x) \psi\left(x, t^{\prime}\right) & =\int_{-\infty}^{\infty} d x \sum_{n} a_{n} X_{m}^{*}(x) X_{n}(x) \\
& =\sum_{n} a_{n} \int_{-\infty}^{\infty} d x X_{m}^{*}(x) X_{n}(x)
\end{aligned}
$$

Before we can evaluate the integral we must prove a condition between the functions $X_{n}$ and $X_{m}$ known as the orthonormal condition

$$
\int_{-\infty}^{\infty} d x X_{m}^{*}(x) X_{n}(x)=\delta_{m n}
$$

where $\delta_{m n}$ is the Kronecker delta defined as

$$
\delta_{m n}=\left\{\begin{array}{l}
1, m=n \\
0, m \neq n
\end{array}\right.
$$

Say we have two functions of position $f(x)$ and $g(x)$ and the Hamiltonian operator $\hat{H}$. The definition of $\hat{H}$ being a Hermitian operator is

$$
\int_{-\infty}^{\infty} d x f(x) \hat{H} g(x)=\int_{-\infty}^{\infty} d x g(x) \hat{H}^{*} f(x) .
$$

If we look at the differential equations

$$
\begin{aligned}
\hat{H} X_{n}(x) & =E_{n} X_{n}(x), \\
\hat{H}^{*} X_{m}^{*}(x) & =E_{m}^{*} X_{m}^{*}(x)
\end{aligned}
$$

and multiply the top equation by $X_{m}^{*}(x)$, the bottom equation by $X_{n}(x)$ and integrate both equations over all $x$ we obtain

$$
\begin{gathered}
\int_{-\infty}^{\infty} d x X_{m}^{*}(x) \hat{H} X_{n}(x)=E_{n} \int_{-\infty}^{\infty} d x X_{m}^{*}(x) X_{n}(x) \\
\int_{-\infty}^{\infty} d x X_{n}(x) \hat{H}^{*} X_{m}^{*}(x)=E_{m}^{*} \int_{-\infty}^{\infty} d x X_{n}(x) X_{m}^{*}(x) .
\end{gathered}
$$


If we subtract the first equation by the second equation we obtain

$$
\int_{-\infty}^{\infty} d x X_{m}^{*}(x) \hat{H} X_{n}(x)-\int_{-\infty}^{\infty} d x X_{n}(x) \hat{H}^{*} X_{m}^{*}(x)=\left(E_{n}-E_{m}^{*}\right) \int_{-\infty}^{\infty} d x X_{m}^{*}(x) X_{n}(x) .
$$

Using the property A.1.16) we get for $m=n$

$$
0=\left(E_{n}-E_{n}^{*}\right) \int_{-\infty}^{\infty} d x X_{n}^{*}(x) X_{n}(x)
$$

and since the integral is non-zero we conclude that the energy levels are real. If $n \neq m$ we get

$$
0=\left(E_{n}-E_{m}\right) \int_{-\infty}^{\infty} d x X_{m}^{*}(x) X_{n}(x)
$$

and since the states are non-degenerate, the energy levels are not equal and the integral must be zero. If $n=m$ the integral

$$
\int_{-\infty}^{\infty} d x X_{n}^{*}(x) X_{n}(x)
$$

is less than infinity and it can be interpreted as the total probability of finding a particle somewhere if it is normalized to

$$
\int_{-\infty}^{\infty} d x X_{n}^{*}(x) X_{n}(x)=1
$$

So A.1.14 holds. Now, we are ready to evaluate A.1.13 by the orthonormality condition of the functions $X_{n}$ and $X_{m}$. The right hand side of A.1.13 is

$$
\sum_{n} a_{n} \int_{-\infty}^{\infty} d x X_{m}^{*}(x) X_{n}(x)=\sum a_{n} \delta_{m n}=a_{m}
$$

since the Kronecker delta, $\delta_{m n}$, kills all the integrals where $m \neq n$. So $a_{m}$ ends up as

$$
a_{m}=\int_{-\infty}^{\infty} d x X_{m}^{*}(x) \psi\left(x, t^{\prime}\right) .
$$

By substitution of $a_{m}$ in A.1.11 we obtain

$$
\psi\left(x^{\prime}, t^{\prime}\right)=\int_{-\infty}^{\infty} d x \sum_{n} X_{n}^{*}(x) X_{n}\left(x^{\prime}\right) \psi\left(x, t^{\prime}\right)
$$

where we have interchanged $x$ and $x^{\prime}$. We see that this is an operation which propagates the wave-function $\psi\left(x^{\prime}, t^{\prime}\right)$ to $\psi\left(x, t^{\prime}\right)$ through the spatial solutions of the Schrödinger equation.

\section{A.2 The Free Particle Propagator}

The most basic propagator must be the propagator for which there are no forces acting upon a particle i.e. a free particle. The Lagrangian for a free particle in one dimension is

$$
L=\frac{1}{2} m \dot{x}^{2}
$$

where $\dot{x}$ is the time derivative of the position. From equation 3.2 .13 we can clearly see that we have a quadratic Lagrangian and all we need to do is to find the classical action of the particle multiply by $i / \hbar$ and exponentiate it apart from some normalization constant. The next thing to do is to find the equations of motion retrieved from the principle of least action that minimizes the action for a system. Thus one will get Lagrange's equations if the boundary remains fixed under an arbitrary variation. The equation of motion from Lagrange's equations is

$$
\frac{d}{d t}(m \dot{x})=0, \quad m \dot{x}=\text { constant }
$$


and if we substitute this in the action $S$ we obtain the classical action $S_{c l}$ as

$$
S_{c l}=\int_{t_{1}}^{t_{2}} d t \frac{1}{2} m \dot{x}^{2}=\left.\frac{1}{2} m \dot{x}^{2} t\right|_{x\left(t_{1}\right)=x_{1}} ^{x\left(t_{2}\right)=x_{2}}=\frac{1}{2} m \frac{\left(x_{2}-x_{1}\right)^{2}}{\left(t_{2}-t_{1}\right)^{2}}\left(t_{2}-t_{1}\right)=\frac{1}{2} m \frac{\left(x_{2}-x_{1}\right)^{2}}{t_{2}-t_{1}}
$$

where we assume that the mass of the particle does not change under its motion. Hence by multiplying this by $i \hbar$ and exponentiate we get the propagator for the free particle as

$$
K\left(x_{2}, t_{2} ; x_{1}, t_{1}\right)=A\left(t_{1}, t_{2}\right) \exp \left\{i S_{c l}\left(x_{2}, x_{1}\right) / \hbar\right\}=A\left(t_{1}, t_{2}\right) \exp \left\{\frac{i m}{2 \hbar} \frac{\left(x_{2}-x_{1}\right)^{2}}{t_{2}-t_{1}}\right\}
$$

where we have multiplied the exponential with and normalization constant $A$ accordingly to 3.2.13. Let us now determine the constant $A$ : First we change variables $t-t_{1}=\epsilon$ then the free propagator can be written as

$$
K\left(x_{2}, \epsilon ; x_{1}, 0\right)=A(\epsilon) \exp \left\{\frac{i m}{2 \hbar} \frac{\left(x_{2}-x_{1}\right)^{2}}{\epsilon}\right\} .
$$

In the limit of $\epsilon \rightarrow 0$ the propagator must reduce to a delta function $\delta\left(x_{2}-x_{1}\right)$ where the delta function is defined here by

$$
\delta\left(x_{2}-x_{1}\right)=\frac{1}{\sqrt{\pi}} \lim _{\epsilon \rightarrow 0}\left[\frac{1}{\sqrt{\epsilon}} \exp \left[-\frac{\left(x_{2}-x_{1}\right)^{2}}{\epsilon}\right]\right] .
$$

Thus by taking the limit as $\epsilon$ goes to zero of the propagator we get

$$
\lim _{\epsilon \rightarrow 0} K\left(x_{2}, \epsilon ; x_{1}, 0\right)=\lim _{\epsilon \rightarrow 0} A(\epsilon)\left\{\exp \left[-\frac{m\left(x_{2}-x_{1}\right)^{2}}{2 i \hbar \epsilon}\right]\right\} .
$$

The next step is to rewrite the just mentioned limit such that it looks like A.2.6. By changing $\epsilon=m \tau / 2 i \hbar$ and introduce factors of $\sqrt{\pi}$ and $\sqrt{\tau}$ we get instead

$$
\lim _{\tau \rightarrow 0} K\left(x_{2}, \tau ; x_{1}, 0\right)=\frac{1}{\sqrt{\pi}} \lim _{\tau \rightarrow 0} A(\tau) \sqrt{\pi \tau}\left\{\frac{1}{\sqrt{\tau}} \exp \left[-\frac{\left(x_{2}-x_{1}\right)^{2}}{\tau}\right]\right\}
$$

and one can easily see that we must choose $A(\tau)$ as

$$
A(\tau)=\sqrt{\frac{1}{\pi \tau}}
$$

If we now change back $\tau=2 i \hbar \epsilon / m=2 i \hbar\left(t_{2}-t_{1}\right) / m$ and substitute for the previously unknown normalization constant $A$ we obtain for the free particle propagator

$$
K\left(x_{2}, t_{2} ; x_{1}, t_{1}\right)=\sqrt{\frac{m}{2 \pi i \hbar\left(t_{2}-t_{1}\right)}} \exp \left\{\frac{i m}{2 \hbar} \frac{\left(x_{2}-x_{1}\right)^{2}}{t_{2}-t_{1}}\right\} .
$$

\section{A.3 The Propagator for the Harmonic Oscillator}

The structure to obtain the propagator for the harmonic oscillator follow exactly the same lines as for the free particle case. From classical mechanics one discovers that the Lagrangian is quadratic in kinetic energy and potential energy. So all we have to consider is finding what equations of motion that minimizes the action and a normalization constant. The Lagrangian for the harmonic oscillator is stated as

$$
L(x(t), \dot{x}(t))=\frac{1}{2} m \dot{x}^{2}-\frac{1}{2} m \omega^{2} x^{2}=\frac{1}{2} m \dot{x}^{2}-\frac{1}{2} k x^{2}
$$


where $k=m \omega^{2}$ and $\omega$ is the frequency of the system. Lagrange's equations give us the equation of motion as

$$
\ddot{x}(t)+\omega^{2} x(t)=0 .
$$

This is a second order differential equation and a solution to this can always be written as

$$
\begin{aligned}
& x(t)=C \sin (\omega t+\alpha) \\
& \dot{x}(t)=\omega C \cos (\omega t+\alpha)
\end{aligned}
$$

where $C$ and $\alpha$ are arbitrary constants. If we substitute A.3.2 in A.3.1 we obtain the classical Lagrangian and by substituting this in the action we obtain the classical action as

$$
S_{c l}[x(t)]=\int_{t_{1}}^{t_{2}} d t\left[\frac{1}{2} m \dot{x}^{2}+\frac{1}{2} m \ddot{x} x\right] .
$$

The integrand can be rewritten using integration by parts as

$$
\begin{aligned}
\frac{1}{2} m \dot{x}^{2}+\frac{1}{2} m \ddot{x} x & =\frac{1}{2} m\left[\frac{d}{d t}(x \dot{x})-x \ddot{x}\right]+\frac{1}{2} m \ddot{x} x \\
& =\frac{1}{2} m \frac{d}{d t}(x \dot{x})
\end{aligned}
$$

and then we can evaluate the classical action as

$$
S_{c l}[x(t)]=\int_{t_{1}}^{t_{2}} d t \frac{1}{2} m \frac{d}{d t}(x \dot{x})=\left.\frac{1}{2} m x(t) \dot{x}(t)\right|_{x\left(t_{1}\right)=x_{1}} ^{x\left(t_{2}\right)=x_{2}}=\frac{1}{2} m\left[x\left(t_{2}\right) \dot{x}\left(t_{2}\right)-x\left(t_{1}\right) \dot{x}\left(t_{1}\right)\right]
$$

where $m$ is again assumed to be constant and the boundaries invariant under a variation. Now we will rewrite the solution A.3.3 and its time derivative as

$$
\begin{aligned}
x(t) & =C \sin \left(\omega\left(t-t_{1,2}\right)+\omega t_{1,2}+\alpha\right) \\
& =C \sin \left(\omega\left(t-t_{1,2}\right)\right) \cos \left(\omega t_{1,2}+\alpha\right)+C \cos \left(\omega\left(t-t_{1,2}\right)\right) \sin \left(\omega t_{1,2}+\alpha\right) . \\
\dot{x}(t) & =C \omega \cos \left(\omega\left(t-t_{1,2}\right)+\omega t_{1,2}+\alpha\right) . \\
& =C \omega \cos \left(\omega\left(t-t_{1,2}\right)\right) \cos \left(\omega t_{1,2}+\alpha\right)-C \omega \sin \left(\omega\left(t-t_{1,2}\right)\right) \sin \left(\omega t_{1,2}+\alpha\right)
\end{aligned}
$$

where we have introduced the times at the endpoints. From this one can easily see that

$$
\begin{aligned}
& x\left(t_{1}\right)=C \sin \left(\omega t_{1}+\alpha\right) \\
& \dot{x}\left(t_{1}\right)=C \omega \cos \left(\omega t_{1}+\alpha\right) \\
& x\left(t_{2}\right)=C \sin \left(\omega t_{2}+\alpha\right) \\
& \dot{x}\left(t_{2}\right)=C \omega \cos \left(\omega t_{2}+\alpha\right)
\end{aligned}
$$

hence the equations in A.3.7 becomes after substituting the above equations

$$
\begin{aligned}
& x(t)=\frac{\dot{x}\left(t_{1,2}\right)}{\omega} \sin \left(\omega\left(t-t_{1,2}\right)\right)+x\left(t_{1,2}\right) \cos \left(\omega\left(t-t_{1,2}\right)\right) \\
& \dot{x}(t)=\dot{x}\left(t_{1,2}\right) \cos \left(\omega\left(t-t_{1,2}\right)\right)-\omega x\left(t_{1,2}\right) \sin \left(\omega\left(t-t_{1,2}\right)\right) .
\end{aligned}
$$

In order to get rid of the time derivatives in A.3.6 we substitute the above equation in the bottom one so we get

$$
\dot{x}(t)=\frac{\omega x(t)-\omega x\left(t_{1,2}\right) \cos \left(\omega\left(t-t_{1,2}\right)\right)}{\sin \left(\omega\left(t-t_{1,2}\right)\right)} \cos \left(\omega\left(t-t_{1,2}\right)\right)-\omega x\left(t_{1,2}\right) \sin \left(\omega\left(t-t_{1,2}\right)\right)
$$


and the products in A.3.6 becomes by substituting the above equations

$$
\begin{aligned}
& x\left(t_{2}\right) \dot{x}\left(t_{2}\right)=\frac{\omega x_{2}^{2}-\omega x_{1} x_{2} \cos \left(\omega\left(t_{2}-t_{1}\right)\right)}{\sin \left(\omega\left(t_{2}-t_{1}\right)\right)} \cos \left(\omega\left(t_{2}-t_{1}\right)\right)-\omega x_{2} x_{1} \sin \left(\omega\left(t_{2}-t_{1}\right)\right) \\
& x\left(t_{1}\right) \dot{x}\left(t_{1}\right)=\frac{-\omega x_{1}^{2}+\omega x_{1} x_{2} \cos \left(\omega\left(t_{2}-t_{1}\right)\right)}{\sin \left(\omega\left(t_{2}-t_{1}\right)\right)} \cos \left(\omega\left(t_{2}-t_{1}\right)\right)+\omega x_{2} x_{1} \sin \left(\omega\left(t_{2}-t_{1}\right)\right) .
\end{aligned}
$$

Thus the classical action is after some manipulation

$$
\begin{aligned}
S_{c l}[x(t)] & =\frac{1}{2} m\left(x\left(t_{2}\right) \dot{x}\left(t_{2}\right)-x\left(t_{1}\right) \dot{x}\left(t_{1}\right)\right) \\
& =\frac{m \omega}{2 \sin \left(\omega\left(t_{2}-t_{1}\right)\right)} \\
& \times\left\{x_{2}^{2} \cos \left(\omega\left(t_{2}-t_{1}\right)\right)-x_{1} x_{2} \cos ^{2}\left(\omega\left(t_{2}-t_{1}\right)\right)-x_{1} x_{2} \sin ^{2}\left(\omega\left(t_{2}-t_{1}\right)\right)\right. \\
& \left.+x_{1}^{2} \cos \left(t_{2}-t_{1}\right)-x_{1} x_{2} \cos ^{2}\left(\omega\left(t_{2}-t_{1}\right)\right)-x_{1} x_{2} \sin ^{2}\left(\omega\left(t_{2}-t_{1}\right)\right)\right\} \\
& =\frac{m \omega}{2 \sin \left(\omega\left(t_{2}-t_{1}\right)\right)}\left\{\left(x_{1}^{2}+x_{2}^{2}\right) \cos \left(\omega\left(t_{2}-t_{1}\right)\right)-2 x_{1} x_{2}\right\}
\end{aligned}
$$

and since the Lagrangian is quadratic we obtain for the propagator

$$
\begin{aligned}
& K\left(x_{2}, t_{2} ; x_{1}, t_{1}\right)= \\
& A\left(t_{2}-t_{1}\right) \exp \left\{\frac{i m \omega}{2 \hbar \sin \left(\omega\left(t_{2}-t_{1}\right)\right)}\left[\left(x_{1}^{2}+x_{2}^{2}\right) \cos \left(\omega\left(t_{2}-t_{1}\right)\right)-2 x_{1} x_{2}\right]\right\} \\
& , \omega\left(t_{2}-t_{1}\right) \neq n \pi, \quad n=0, \pm 1 \pm 2, \cdots
\end{aligned}
$$

Next step is to determine the normalization constant $A$. We know that in the limit of $\omega \rightarrow 0$ the propagator must reduce to the free particle propagator. But because of the cosine in the exponential it is not obvious if we can rewrite it as to resemble the delta function we defined earlier, if even possible. So we follow a different approach to find the normalization constant: First let us change $t-t_{1}=T$. Then the propagator is again

$$
K\left(x_{2}, T ; x_{1}, 0\right)=A(T) \exp \left\{\frac{i m \omega}{2 \hbar \sin (\omega T)}\left[\left(x_{1}^{2}+x_{2}^{2}\right) \cos (\omega T)-2 x_{1} x_{2}\right]\right\} .
$$

Next we use the property 3.5 .10$)$ to write

$$
\delta\left(x_{1}-x_{1}^{\prime}\right)=\int_{-\infty}^{\infty} d x K^{*}\left(x, T ; x_{1}^{\prime}, 0\right) K\left(x, T ; x_{1}, 0\right)
$$

where the propagator and its complex conjugate are given by

$$
\begin{aligned}
K^{*}\left(x, T ; x_{1}^{\prime}, 0\right) & =A(T)^{*} \exp \left[-i S_{c l}\left(x, T ; x_{1}^{\prime}, 0\right) / \hbar\right], \\
K\left(x, T ; x_{1}, 0\right) & =A(T) \exp \left[i S_{c l}\left(x, T ; x_{1}, 0\right) / \hbar\right] .
\end{aligned}
$$

By substituting the explicit forms back in A.3.15 we get

$$
\begin{aligned}
\delta\left(x_{1}-x_{1}^{\prime}\right) & =|A(T)|^{2} \int_{-\infty}^{\infty} d x \exp \left\{i\left[S_{c l}\left(x, T ; x_{1}, 0\right)-S_{c l}\left(x, T ; x_{1}^{\prime}, 0\right)\right] / \hbar\right\} \\
& =|A(T)|^{2} \int_{-\infty}^{\infty} d x \exp \left\{\frac{i}{\hbar} \frac{\partial S_{c l}^{\prime}}{\partial\left(x_{1}-x_{1}^{\prime}\right)}\left(x_{1}-x_{1}^{\prime}\right)\right\}
\end{aligned}
$$


where we have expanded the different actions to a first order in the difference of the positions and where

$$
S_{c l}^{\prime}=S_{c l}\left(x, T ; x_{1}, 0\right)-S_{c l}\left(x, T ; x_{1}^{\prime}, 0\right)=\frac{m \omega}{2 \sin (\omega T)}\left\{\left(x_{1}^{2}-x_{1}^{\prime 2}\right) \cos (\omega T)-2 x\left(x_{1}-x_{1}^{\prime}\right)\right\} .
$$

The partial derivative in the exponential is

$$
\begin{aligned}
\frac{\partial S_{c l}^{\prime}}{\partial\left(x_{1}-x_{1}^{\prime}\right)} & =\frac{\partial}{\partial\left(x_{1}-x_{1}^{\prime}\right)}\left[\frac{m \omega}{2 \sin (\omega T)}\left\{\left(x_{1}^{2}-x_{1}^{\prime 2}\right) \cos (\omega T)-2 x\left(x_{1}-x_{1}^{\prime}\right)\right\}\right] \\
& =-\frac{m \omega x}{\sin (\omega T)}+\frac{m \omega \cos (\omega t)}{2 \sin (\omega t)}\left(x_{1}+x_{1}^{\prime}\right)
\end{aligned}
$$

and if we substitute this in A.3.15 we get

$$
\delta\left(x_{1}-x_{1}^{\prime}\right)=|A(T)|^{2} \exp \left\{\frac{i m \omega \cos (\omega t)}{2 \sin (\omega t)}\left(x_{1}^{2}-x_{1}^{\prime 2}\right)\right\} \int_{-\infty}^{\infty} d x \exp \left\{-\frac{i m \omega x}{\hbar} \frac{\left(x_{1}-x_{1}^{\prime}\right)}{\sin (\omega T)}\right\} .
$$

A change of variable $y=-m \omega x / \hbar \sin (\omega T)$ gives

$$
\begin{aligned}
\delta\left(x_{1}-x_{1}^{\prime}\right) & =|A(T)|^{2} \exp \left\{\frac{i m \omega \cos (\omega t)}{2 \sin (\omega t)}\left(x_{1}^{2}-x_{1}^{\prime 2}\right)\right\} \frac{\hbar \sin (\omega T)}{m \omega} \int_{-\infty}^{\infty} d y \exp \left[i y\left(x_{1}-x_{1}^{\prime}\right)\right] \\
& =2 \pi|A(T)|^{2} \exp \left\{\frac{i m \omega \cos (\omega t)}{2 \sin (\omega t)}\left(x_{1}^{2}-x_{1}^{\prime 2}\right)\right\} \frac{\hbar \sin (\omega T)}{m \omega} \delta\left(x_{1}-x_{1}^{\prime}\right) \\
& =2 \pi|A(T)|^{2} \frac{\hbar \sin (\omega T)}{m \omega} \delta\left(x_{1}-x_{1}^{\prime}\right)
\end{aligned}
$$

where we have used another definition of the Delta function. By dropping the delta functions on both sides we have

$$
A(T)=\sqrt{\frac{m \omega}{2 \pi \hbar \sin (\omega T)}}
$$

with an undetermined phase. This phase can however be determined by knowing that the harmonic oscillator propagator must reduce to the free particle propagator when $\omega T \rightarrow 0$. Hence the phase we are looking for is $1 / \sqrt{i}$ and with this the propagator becomes

$$
\begin{aligned}
& K\left(x_{2}, t_{2} ; x_{1}, t_{1}\right)= \\
& \quad \sqrt{\frac{m \omega}{2 \pi i \hbar \sin \left(\omega\left(t_{2}-t_{1}\right)\right)}} \exp \left\{\frac{i m \omega}{2 \hbar \sin \left(\omega\left(t_{2}-t_{1}\right)\right)}\left[\left(x_{1}^{2}+x_{2}^{2}\right) \cos \left(\omega\left(t_{2}-t_{1}\right)\right)-2 x_{2} x_{1}\right]\right\} \\
& \omega\left(t_{2}-t_{1}\right) \neq n \pi .
\end{aligned}
$$

\section{A.4 The Propagator for an Entangled Path with the Origin Removed}

When we derived the previous propagators we dealt with Lagrangians that were quadratic in position and velocity. The classical action obtained from the classical equations of motion could simply be exponentiated and the propagator be derived. In this section we are going to begin with the propagator for a free system and introduce certain restriction of its motion. We are also extending the space to three dimensions and the configuration acquires cylindrical symmetry so we are working in the plane. Since we are working in a plane the choice of coordinates is $(r, \theta)$. In the $(r, \theta)$ plane we have two vectors $\boldsymbol{r}_{1}$ and $\boldsymbol{r}_{2}$ where each of these vectors can be described by the angles $\theta_{1}$ and $\theta_{2}$ measured from an origo at $\boldsymbol{r}=\mathbf{0}$ and lengths $r_{1}=\left|\boldsymbol{r}_{1}\right|$ and $r_{2}=\left|\boldsymbol{r}_{2}\right|$ with it. When the particle moves from $\boldsymbol{r}_{1}$ to $\boldsymbol{r}_{2}$ we must consider all possible ways a system can move from one state to another because of the principle of superposition of states. We are also going 


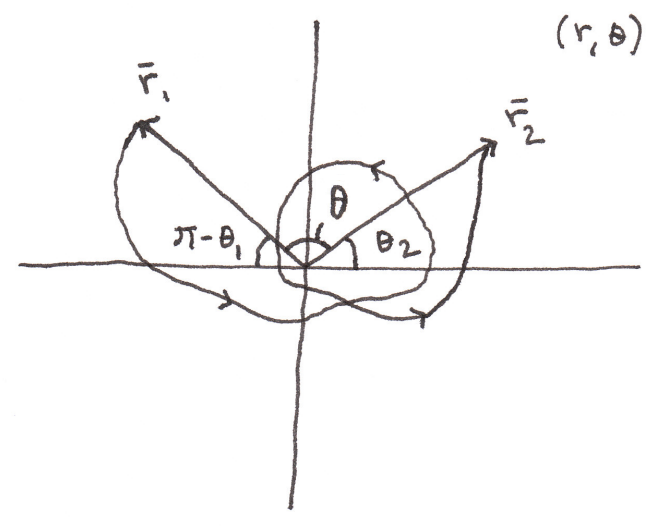

Figure 6: A simplified view of the propagation from a state at $\left(\boldsymbol{r}_{1}, t_{1}\right)$ to a state at $\left(\boldsymbol{r}_{2}, t_{2}\right)$. The propagation is in a counterclockwise direction but might as well be in a clockwise direction.

to remove the origin so that the system may not ever be in a state at that point and allow the particles encircle the origin any way they like.

This ensures the restriction of the angles as

$$
\int_{t_{1}}^{t_{2}} d t \frac{d \theta}{d t}=\Theta+2 \pi n, \quad n=0, \pm 1, \pm 2, \cdots
$$

where the limits of integration have been changed from the angles to the times. Instead of this restriction suppose we write the right hand side as

$$
\int_{t_{1}}^{t_{2}} d t \dot{\theta}=\phi
$$

where $\phi$ is a restriction on the angles we wish to show is equal to $\Theta+2 \pi n$. For a free particle in three dimensional space plus time the propagator is simply just

$$
K\left(\boldsymbol{r}_{2}, t_{2} ; \boldsymbol{r}_{1}, t_{1}\right) \equiv K=\int_{\boldsymbol{r}\left(t_{1}\right)=\boldsymbol{r}_{1}}^{\boldsymbol{r}\left(t_{2}\right)=\boldsymbol{r}_{2}} \mathcal{D} \boldsymbol{r}(t) \exp (i S[\boldsymbol{r}(t)] / \hbar)
$$

where we have defined a shorthand for the propagator as $K$. Under the restriction A.4.2 what operation selects out only the contributing probability amplitudes to the propagator $K$ ? The Dirac delta function have just that property that when integrated selects out the contributing terms to the integral. So by our new restriction the propagator can be written as

$$
K_{\phi}=\int \mathcal{D} \boldsymbol{r}(t) \delta\left(\phi-\int_{t_{1}}^{t_{2}} d t \dot{\theta}\right) \exp (i S[\boldsymbol{r}(t)] / \hbar)
$$

where the subscript on the left hand side indicates that the propagator depends on what we choose $\phi$ as. But from a integral definition of the Delta function

$$
\delta\left(\int_{t_{1}}^{t_{2}} d t \dot{\theta}-\phi\right)=\frac{1}{2 \pi} \int_{-\infty}^{\infty} d \lambda \exp \left\{i \lambda\left(\phi-\int_{t_{1}}^{t_{2}} d t \dot{\theta}\right)\right\} .
$$

we get the $\phi$ dependent propagator as

$$
K_{\phi}=\frac{1}{2 \pi} \int_{-\infty}^{\infty} d \lambda K_{\lambda} \exp \{i \lambda \phi\}
$$

that need to be integrated over all $\phi$ to get the contributing terms that constructs $K$ and $K_{\lambda}$ is defined as

$$
K_{\lambda}=\int_{\boldsymbol{r}_{1}}^{\boldsymbol{r}_{2}} \mathcal{D} \boldsymbol{r}(t) \exp \left\{\frac{i}{\hbar} S[\boldsymbol{r}(t)]-i \lambda \int_{t_{1}}^{t_{2}} d t \dot{\theta}\right\} .
$$


The action for the free particle can be explicitly written as

$$
S[\boldsymbol{r}(t)]=S_{0}[\boldsymbol{r}(t)]=\int_{t_{1}}^{t_{2}} d t \frac{1}{2} m \dot{\boldsymbol{r}}^{2} .
$$

where $\dot{\boldsymbol{r}}$ is the velocity of the radial vector $\boldsymbol{r}$. If we substitute the explicit action in the exponential of $K_{\lambda}$ we obtain

$$
\exp \left\{\frac{i}{\hbar} \int_{t_{1}}^{t_{2}} d t \frac{1}{2} m \dot{r}^{2}-i \lambda \int_{t_{1}}^{t_{2}} d t \dot{\theta}\right\}=\exp \left\{\frac{i}{\hbar} \int_{t_{1}}^{t_{2}} d t\left(\frac{1}{2} m \dot{r}^{2}-\hbar \lambda \dot{\theta}\right)\right\} .
$$

If we define

$$
S^{\prime}[\boldsymbol{r}(\boldsymbol{t})]=S[\boldsymbol{r}(t)]_{0}-\int_{t_{1}}^{t_{2}} d t \hbar \lambda \dot{\theta}
$$

where $S_{0}$ is the free particle action we see that the free particle action has been reduced by a term with units of action. The idea of resolving the explicit form of the propagator $K_{\lambda}$ is very easy in principle but the mathematics leading to result is painstakingly difficult as will be clear as we progress. We begin by following the time-slicing method since the Lagrangian is not simply quadratic in position and velocity as it seems by the following: For small times $\epsilon$, the action can be resolved in discrete parts in number of $N=\left(t_{2}-t_{1}\right) / \epsilon$. The effective Lagrangian is therefore discretized as

$$
L^{\prime}=L^{\prime}\left(\frac{\theta_{j}-\theta_{j-1}}{\epsilon}, \frac{\boldsymbol{r}_{j}-\boldsymbol{r}_{j-1}}{\epsilon}, \frac{r_{j}+r_{j-1}}{2}\right)
$$

or explicitly

$$
L^{\prime}=\frac{m}{2}\left(\frac{\boldsymbol{r}_{j}-\boldsymbol{r}_{j-1}}{\epsilon}\right)^{2}-\hbar \lambda \frac{\left(\theta_{i}-\theta_{j-1}\right)}{\epsilon}
$$

$K_{\lambda}$ can now be written as

$$
K_{\lambda}=\lim _{N \rightarrow \infty} C_{N} \int \cdots \int \prod_{j=1}^{N-1} d \boldsymbol{r}_{j} \exp \left\{\frac{i}{\hbar} \sum_{j=1}^{N} S\left[\boldsymbol{r}_{j}, \boldsymbol{r}_{j-1}\right]\right\} .
$$

where $C_{N}$ is a normalization constant,

$$
S\left[\boldsymbol{r}_{j}, \boldsymbol{r}_{j-1}\right] \equiv \frac{m}{2 \epsilon}\left(\Delta \boldsymbol{r}_{j}\right)^{2}-\hbar \lambda \Delta \theta_{j}
$$

is the discrete partial action and the averages as $\Delta \boldsymbol{r}_{j} \equiv \boldsymbol{r}_{j}-\boldsymbol{r}_{j-1}$ and $\Delta \theta_{j} \equiv \theta_{j}-\theta_{j-1}$. Since we are working in polar coordinates

$$
\left(\Delta r_{j}\right)^{2}=r_{j}^{2}+r_{j-1}^{2}-2 r_{j} r_{j-1} \cos \left(\theta_{j}-\theta_{j-1}\right)
$$

and (A.4.14 can therefore be written as

$$
S\left[\boldsymbol{r}_{j}, \boldsymbol{r}_{j-1}\right]=\frac{m}{2 \epsilon}\left(r_{j}^{2}+r_{j-1}^{2}\right)-\frac{m}{\epsilon} r_{j} r_{j-1} \cos \left(\theta_{j}-\theta_{j-1}\right)-\hbar \lambda\left(\theta_{j}-\theta_{j-1}\right)
$$

which clearly show that the Lagrangian is non-linear. The main goal is to separate the partial action such that we can integrate over each variable independently and resolve any cross terms. There are some non-trivial tricks that are used but will be explained as we move along. We begin by resolving the cosine term. It can be found inside a Taylor expansion of a cosine with a undetermined constant term $a$ as

$$
\cos \left(\Delta \theta_{j}+a \epsilon\right)=\cos \left(\Delta \theta_{j}\right)-a \epsilon \Delta \theta_{j}-\frac{1}{2} a^{2} \epsilon^{2}
$$


where we have terminated the series after second order in $\epsilon$. If we now compare this to the partial action we can identify two terms that exists within the expansion so let us write the expansion in a more suggestive way so to resemble the terms in the discrete action as

$$
\frac{m}{\epsilon} r_{j} r_{j-1} \cos \left(\Delta \theta_{j}+a \epsilon\right)+\frac{m r_{j} r_{j-1}}{2} \epsilon a^{2}=\frac{m}{\epsilon} r_{j} r_{j-1} \cos \left(\Delta \theta_{j}\right)-a m r_{j} r_{j-1} \Delta \theta_{j} .
$$

where each term has been multiplied by a factor of $\left(m r_{j} r_{j-1}\right) / \epsilon$. So we can identify $a$ as

$$
a=\frac{-\hbar \lambda}{m r_{j} r_{j-1}}
$$

and by substituting the left hand side of $\mathrm{A.4.18}$ in the partial action we obtain

$$
S\left[\boldsymbol{r}_{j}, \boldsymbol{r}_{j-1}\right]=\frac{m}{2 \epsilon}\left(r_{j}^{2}+r_{j-1}^{2}\right)-\frac{m}{\epsilon} r_{j} r_{j-1} \cos \left(\Delta \theta_{j}-\frac{\hbar \lambda \epsilon}{m r_{j} r_{j-1}}\right)-\frac{\epsilon \hbar^{2} \lambda^{2}}{2 m r_{j} r_{j-1}} .
$$

So the cross term with an angular and radial term is resolved but now we have introduced a cosine that has both angular and radial dependence. This term when exponentiated is

$$
\exp \left\{-\frac{i m r_{j} r_{j-1}}{\hbar \epsilon} \cos \left(\Delta \theta_{j}-\frac{\hbar \lambda \epsilon}{m r_{j} r_{j-1}}\right)\right\}
$$

The exponential can be expanded in a modified Bessel function of an asymptotic form for small $\epsilon$ as 11

$$
\exp \left(\frac{u}{\epsilon} \cos (z)\right)=\sum_{l_{j}=-\infty}^{\infty} \exp \left(i l_{j} z\right) I_{l_{j}}\left(\frac{u}{\epsilon}\right)
$$

where $\left(-i m r_{j} r_{j-1}\right) / \hbar=u, z=\Delta \theta_{j}-(\lambda \epsilon) / i u$ and $I$ is the asymptotic modified Bessel function. The Bessel function can be expanded approximately as

$$
I_{l_{j}}\left(\frac{u}{\epsilon}\right) \approx\left(\frac{\epsilon}{2 \pi u}\right)^{1 / 2} \exp \left\{\frac{u}{\epsilon}-\frac{1}{2}\left(l_{j}^{2}-\frac{1}{4}\right) \frac{\epsilon}{u}+\mathcal{O}\left(\epsilon^{2}\right)\right\}
$$

where $\mathcal{O}$ is the big-o notation collecting terms higher than second order in $\epsilon$. By adding the last term, quadratic in $\lambda$, from equation A.4.20 and the linear term in $\lambda$, from the definition of $z$, to the expansion of the asymptotic modified Bessel function we get after some algebra

$$
\begin{aligned}
& \sum_{l_{j}=-\infty}^{\infty} \exp \left(i l_{j} \Delta \theta_{j}\right)\left(\frac{\epsilon}{2 \pi u}\right)^{1 / 2} \exp \left(\frac{u}{\epsilon}-\frac{1}{2}\left[\left(l_{j}+\lambda\right)^{2}-\frac{1}{4}\right] \frac{\epsilon}{u}+\mathcal{O}\left(\epsilon^{2}\right)\right) \\
= & \sum_{l_{j}=-\infty}^{\infty} \exp \left(i l_{j} \Delta \theta_{j}\right) I_{l_{j}+\lambda}\left(\frac{u}{\epsilon}\right) .
\end{aligned}
$$

where we have changed the lower index of the modified Bessel function to $l_{j}+\lambda$ with no loss since if this expansion of the Bessel function were in fact not equal in terms higher than first order in $\epsilon$ in A.4.23 the terms of second order in $\epsilon$ and higher would be neglected anyways and the new form can be contracted in the limit of $\epsilon \rightarrow 0$. The complete exponential of the discretized action is now

$$
\exp \left\{\frac{i}{\hbar} \sum_{j=1}^{N} S\left[\boldsymbol{r}_{j}, \boldsymbol{r}_{j-1}\right]\right\}=\prod_{j=1}^{N} \sum_{l_{j}=-\infty}^{\infty} \exp \left(i l_{j} \Delta \theta_{j}\right) \mathcal{R}_{l_{j}+\lambda}\left(r_{j}, r_{j-1}\right)
$$

where we have defined the radial function

$$
\mathcal{R}_{l_{j}-\lambda}=\exp \left\{\frac{i m}{2 \hbar \epsilon}\left(r_{j}^{2}+r_{j-1}^{2}\right)\right\} I_{l_{j}+\lambda}\left(\frac{-i m r_{j} r_{j-1}}{\hbar \epsilon}\right) .
$$

\footnotetext{
${ }^{11}$ doi: $10.1063 / 1.523588$, p. 2319
} 
In equation A.4.25 we can interchange the summation and product and by substituting the result in A.4.13 with $d \boldsymbol{r}_{\boldsymbol{j}}=r_{j} d r_{j} d \theta_{j}$ we obtain for $K_{\lambda}$

$$
\begin{aligned}
K_{\lambda}\left(\boldsymbol{r}_{2}, t_{2} ; \boldsymbol{r}_{1}, t_{1}\right) \equiv K_{\lambda}=\lim _{N \rightarrow \infty} C_{N} \sum_{l_{j}=-\infty}^{\infty} \int \cdots \int & \prod_{j=1}^{N-1} r_{j} d r_{j} d \theta_{j} \prod_{j=1}^{N} \\
& \times \exp \left(i l_{j} \Delta \theta_{j}\right) \mathcal{R}_{l_{j}+\lambda}\left(r_{j}, r_{j-1}\right) .
\end{aligned}
$$

At this point we have successfully separated the angular and radial terms. What we see here is a convolution of angular and radial integrals respectively and we begin by resolving the angular integrals: Let us define the resulting number after we have solved all angular integrals as

$$
\Omega \equiv \int \cdots \int \prod_{j=1}^{N-1} d \theta_{j} \prod_{j=1}^{N} \exp \left[i l_{j}\left(\theta_{j}-\theta_{j-1}\right)\right]
$$

Since per definition of the integral representation of the Kronecker delta

$$
\int d \theta \exp \left[i\left(l_{j}-l_{j}^{\prime}\right) \theta\right]=2 \pi \delta_{l_{j} l_{j}^{\prime}}
$$

we can solve $\Omega$ by integrate the convoluting integrals up to the $N-1$ term and the result of $\Omega$ is

$$
\Omega=(2 \pi)^{N-1} \exp \left[i\left(\theta_{2}-\theta_{1}\right) l_{N}\right]
$$

where we have changed the angular endpoints as $\theta_{N}=\theta_{2}$ and $\theta_{0}=\theta_{1}$. By substituting this in $K_{\lambda}$ we obtain

$$
\begin{aligned}
K_{\lambda}= & \lim _{N \rightarrow \infty} C_{N} \sum_{l_{j}=-\infty}^{\infty}(2 \pi)^{N-1} \exp \left[i\left(\theta_{2}-\theta_{1}\right) l_{j}\right] \\
& \times \int \cdots \int \prod_{j=1}^{N-1} r_{j} d r_{j} \prod_{j=1}^{N} \mathcal{R}_{l_{j}-\lambda}\left(r_{j}, r_{j-1}\right) \\
& =\sum_{l_{j}=-\infty}^{\infty} \exp \left[i\left(\theta_{2}-\theta_{1}\right) l_{j}\right] Q_{l_{j}+\lambda}\left(r_{2}, r_{1}\right)
\end{aligned}
$$

where we have defined the $Q$-function as

$$
Q_{l_{j}-\lambda}\left(r_{2}, r_{1}\right) \equiv \lim _{N \rightarrow \infty}(2 \pi)^{N-1} C_{N} \int \cdots \int \prod_{j=1}^{N-1} r_{j} d r_{j} \prod_{j=1}^{N} \mathcal{R}_{l_{j}+\lambda}\left(r_{j}, r_{j-1}\right) .
$$

Then A.4.6 becomes by substitution of $K_{\lambda}$ as

$$
\begin{aligned}
K_{\phi}\left(\boldsymbol{r}_{2}, t_{2} ; \boldsymbol{r}_{1}, t_{1}\right) & =(2 \pi)^{-1} \int_{-\infty}^{\infty} d \lambda \exp (i \phi \lambda) K_{\lambda} \\
& =(2 \pi)^{-1} \int_{-\infty}^{\infty} d \lambda \exp (i \phi \lambda) \sum_{l_{j}=-\infty}^{\infty} \exp \left(i\left(\theta_{2}-\theta_{1}\right) l_{j}\right) Q_{l_{j}+\lambda}\left(r_{2}, r_{1}\right) \\
& =(2 \pi)^{-1} \int_{-\infty}^{\infty} d \lambda \sum_{l_{j}=-\infty}^{\infty} \exp \left[i\left(\theta_{2}-\theta_{1}\right) l_{j}+i \phi \lambda\right] Q_{l_{j}+\lambda}\left(r_{2}, r_{1}\right) .
\end{aligned}
$$

A shift of lambda, $\lambda \rightarrow l_{j}-\lambda$ gives instead

$$
K_{\phi}\left(\boldsymbol{r}_{2}, t_{2} ; \boldsymbol{r}_{1}, t_{1}\right)=(2 \pi)^{-1} \int_{-\infty}^{\infty} d \lambda \sum_{l_{j}=-\infty}^{\infty} \exp \left[i\left(\theta_{2}-\theta_{1}+\phi\right) l_{j}-i \phi \lambda\right] Q_{\lambda}\left(r_{2}, r_{1}\right)
$$


This can with the help of the identity

$$
\sum_{l_{j}=-\infty}^{\infty} \exp \left[i\left(\theta_{2}-\theta_{1}+\phi\right) l_{j}\right]=2 \pi \sum_{n=-\infty}^{\infty} \delta\left(\theta_{2}-\theta_{1}+\phi+2 \pi n\right)
$$

be written as

$$
K_{\phi}\left(\boldsymbol{r}_{2}, t_{2} ; \boldsymbol{r}_{1}, t_{1}\right)=\sum_{n=-\infty}^{\infty} \delta\left(\theta_{2}-\theta_{1}+\phi+2 \pi n\right) \int_{-\infty}^{\infty} d \lambda \exp (-i \phi \lambda) Q_{\lambda}\left(r_{2}, r_{1}\right)
$$

The angular part has now been sorted out therefore we shall solve for the radial integrals. Now A.4.32 with $\mathcal{R}$ explicitly shown is

$$
\begin{aligned}
Q_{\lambda}\left(r_{2}, r_{1}\right) & =\lim _{N \rightarrow \infty}(2 \pi)^{N-1} C_{N} \int \cdots \int \prod_{j=1}^{N-1} r_{j} d r_{j} \prod_{j=1}^{N} \exp \left\{\frac{i m}{2 \hbar \epsilon}\left(r_{j}^{2}+r_{j-1}^{2}\right)\right\} \\
& \times I_{\lambda}\left(\frac{-i m r_{j} r_{j-1}}{\hbar \epsilon}\right) .
\end{aligned}
$$

The problem now is how to integrate over the convoluted Bessel functions and there exist a paper where just this type of integral is proved to have a closed form 12 . From this paper we can write the general form of the convoluted integrals as

$$
\begin{aligned}
& \int_{0}^{\infty} \cdots \int_{0}^{\infty} \prod_{j=1}^{N-1} r_{j} d r_{j} \exp \left\{i \alpha \sum_{j=1}^{N-1} r_{j}^{2}\right\} \prod_{j=1}^{N} I_{v}\left(-i B r_{j-1} r_{j}\right) \\
& =\prod_{j=1}^{N-1}\left(\frac{i}{2 A_{j}}\right) \exp \left\{-i\left[r_{0}^{2} \sum_{j=1}^{N-1} \frac{B^{2}}{4 A_{j}}+\frac{r_{N}^{2} B^{2}}{A_{N}}\right]\right\} I_{v}\left(-i B_{N} r_{0} r_{N}\right)
\end{aligned}
$$

where $A_{j}, A_{N} B_{N}$ and $B$ are constants left to be determined by the iterated equations

$$
\begin{aligned}
& A_{1}=A, \quad A_{j+1}=A-\frac{B^{2}}{4 A_{j}}, \\
& B_{1}=B, \quad B_{j+1}=B \prod_{k=1}^{j} \frac{B}{2 A_{k}} .
\end{aligned}
$$

We have a freedom to choose the form of the final result to imitate the form of $Q_{\lambda}$ but with the cost of introducing two functions $f_{N}$ and $g_{N}$ such that A.4.38 will be equal to

$$
a_{N} \exp \left(i f_{N} r_{0}^{2}+i g_{N} r_{N}^{2}\right) I_{v}\left(-i B_{N} r_{0} r_{N}\right) \text {. }
$$

If we compare this to $Q_{\lambda}$ then $Q_{\lambda}$ will contain two extra terms

$$
\exp \left\{\frac{i m}{2 \hbar \epsilon}\left(r_{N}^{2}+r_{0}^{2}\right)\right\}
$$

that has not been taken into account in A.4.38 and it is understood that $r_{N}=r_{2}$ and $r_{0}=r_{1}$. If we expand A.4.37 we see that all the intermediate points, between $r_{1}$ and $r_{2}$, appear twice which in fact is accounted for in A.4.38. By identifying the constants $B, f_{N}, g_{N}$ and $a_{N}$ in A.4.38 we obtain the complementing equations as

$$
\begin{aligned}
a_{N}=\prod_{k=1}^{j}\left(\frac{i}{2 A_{j}}\right), \quad f_{N} & =\frac{1}{2} B-\sum_{j=1}^{N-1} \frac{B_{j}^{2}}{4 A_{j}}, \\
g_{N} & =\frac{1}{2} B-\frac{B^{2}}{A_{N}}
\end{aligned}
$$

\footnotetext{
${ }^{12}$ Peak, Inomata, doi: 10.1063/1.1664984
} 
and $B / 2$-terms have been added for the presence of the endpoints missing out in A.4.38 and these equations let us determine the unknowns. It is evident that $A=B$ hence we can expand the terms of $A_{j+1}$ in A.4.39 and obtain

$$
\begin{aligned}
& A_{2}=A-\frac{1}{4} A_{1}=\frac{2+1}{4} A, \\
& A_{3}=A-\frac{1}{3} A_{2}=\frac{3+1}{6} A, \\
& A_{4}=A-\frac{3}{5} A_{3}=\frac{4+1}{8} A, \\
& A_{5}=A-\frac{2}{5} A_{4}=\frac{5+1}{10} A,
\end{aligned}
$$

From this it easy to see that

$$
A_{j}=\frac{j+1}{2 j} A
$$

and by using this result in the equation for $A_{j+1}$ found in $\mathrm{A} .4 .39$ gives

$$
B_{j}=A \prod_{k=1}^{j-1} \frac{A}{2} \frac{2 k}{A(k+1)}=\frac{(j-1) !}{j !} A=\frac{A}{j}
$$

Then by substituting the expressions for $A_{j}$ and $B_{j}$ in A.4.43 we obtain for $f_{N}, g_{N}$ and $a_{N}$ :

$$
\begin{aligned}
f_{N} & =\frac{1}{2} A-\frac{1}{4} \sum_{j=1}^{N-1} \frac{A^{2}}{j^{2}} \frac{2 j}{A(j+1)} \\
& =\frac{1}{2} A-\frac{1}{2} A \sum_{j=1}^{N-1} \frac{1}{j(j+1)}=\frac{1}{2} A-\frac{1}{2} A\left(1-\frac{1}{N}\right)=\frac{A}{2 N} \\
g_{N} & =\frac{1}{2} A-\frac{A^{2}}{4 A_{N}}=\frac{1}{2} A-\frac{1}{2} A \frac{N}{N+1}=\frac{1}{2} A\left(\frac{1}{N+1}\right) \\
a_{N} & =\prod_{j=1}^{N-1}\left(\frac{i}{2 A_{j}}\right)=\prod_{j=1}^{N-1} \frac{i}{2 A} \frac{2 j}{j+1}=\left(\frac{i}{A}\right)^{N-1} \frac{1}{N} .
\end{aligned}
$$

$g_{N}$ can be approximated by substituting $N+1 \approx N$ for large $N$ and by substituting $f_{N}, g_{N}, a_{N}$ into A.4.41 we get

$$
a_{N} \exp \left(i f_{N} r_{0}^{2}+i g_{N} r_{N}^{2}\right) I_{v}\left(-i B_{N} r_{0} r_{N}\right)=\left(\frac{i \hbar \epsilon}{m}\right)^{N-1} \frac{1}{N} \exp \left(\frac{i m}{2 \hbar \tau}\left(r_{2}^{2}+r_{1}^{2}\right)\right)
$$

where we have defined $\tau=N \epsilon=t_{2}-t_{1}$ and identified $r_{N}=r_{2}$ and $r_{0}=r_{1}$. If we substitute this in A.4.38 one should get for $Q_{v}$

$$
\begin{aligned}
Q_{v}\left(r_{2}, \tau ; r_{1}, 0\right) & =\lim _{N \rightarrow \infty} C_{N}\left(\frac{i \hbar \epsilon}{m}\right)^{N-1} N^{-1}(2 \pi)^{N-1} \exp \left\{\frac{i m}{2 \hbar \tau}\left(r_{2}^{2}+r_{1}^{2}\right)\right\} I_{v}\left(\frac{-i m r_{1} r_{2}}{\hbar \tau}\right) \\
& =\lim _{N \rightarrow \infty} C_{N}\left(\frac{2 \pi i \hbar \epsilon}{m}\right)^{N}\left(\frac{m}{2 \pi i \hbar \tau}\right) \exp \left\{\frac{i m}{2 \hbar \tau}\left(r_{2}^{2}+r_{1}^{2}\right)\right\} I_{v}\left(\frac{-i m r_{1} r_{2}}{\hbar \tau}\right) .
\end{aligned}
$$

For $Q_{v}$ to be finite in the limit $N \rightarrow \infty$, the normalization constant must be

$$
C_{N}=\left(\frac{m}{2 \pi i \hbar \epsilon}\right)^{-N}
$$


and the path integral will be defined. Finally we obtain by changing $v=\lambda$ the explicit expression for $Q_{\lambda}$ as

$$
Q_{\lambda}\left(r_{2}, \tau ; r_{1}, 0\right)=\frac{m}{2 \pi i \hbar \tau} \exp \left\{\frac{i m}{2 \hbar \tau}\left(r_{2}^{2}+r_{1}^{2}\right)\right\} I_{\lambda}\left(\frac{-i m r_{1} r_{2}}{\hbar \tau}\right) .
$$

Then A.4.36 becomes

$$
\begin{aligned}
K_{\phi}\left(\boldsymbol{r}_{2}, t_{2} ; \boldsymbol{r}_{1}, t_{1}\right) & =\frac{m}{2 \pi i \hbar\left(t_{2}-t_{1}\right)} \sum_{n=-\infty}^{\infty} \delta\left(\theta_{2}-\theta_{1}+\phi+2 \pi n\right) \\
& \times \exp \left\{\frac{i m}{2 \hbar} \frac{\left(r_{2}^{2}+r_{1}^{2}\right)}{t_{2}-t_{1}}\right\} \int_{-\infty}^{\infty} d \lambda \exp (-i \phi \lambda) I_{\lambda}\left(\frac{-i m r_{1} r_{2}}{\hbar\left(t_{2}-t_{1}\right)}\right)
\end{aligned}
$$

and we obtain $K$ by integrating $K_{\phi}$ over all configurations $\phi$. So far we have use relatively easy methods to separate the radial parts from the angular parts and the result we have obtained for $K_{\phi}$ is very appealing but the integral over a Bessel function remains to be resolved. In the Aharonov-Bohm effect we use this result and solve the integral by limiting it in a semi-classical way by neglecting all terms linear in $\hbar$. The exact solution to this integral remains still unknown however and it may not even be possible to find a closed form. 


\section{References}

[1] D. Peak A. Inomata. Summation over feynman histories in polar coordinates. Journal of Mathematical Physics, pages 1422-1428, 1969. doi: 10.1063/1.1664984.

[2] Vijay A. Singh A. Inomata. Path integrals with a periodic constraint: Entangled strings. Journal of Mathematical Physics, pages 2318-2323, 1978. doi: 10.1063/1.523588.

[3] David Bohm. Quantum Theory. Dover Publications, 1989.

[4] David Chandler. Introduction to Modern Statistical Mechanics. Oxford University Press, 1987.

[5] P. A. M. Dirac. Quantum Mechanics, Third Edition. Oxford, 1947.

[6] Richard P. Feynman. Principles of Least Action in Quantum Mechanics. PhD thesis, Princeton, 1942.

[7] Albert R. Hibbs Richard P. Feynman. Quantum Mechanics and Path Integrals, Emended Edition. Dover, 2010.

[8] L.S. Schulman. Techniques and Applications of Path Integration, Second Edition. Dover, 2005.

[9] M. Reuter W. Dittrich. Classical and Quantum Dynamics, Second Edition. Springer, 1996. 